\title{
The Effects of Model Misspecification When Fitting Generalized Linear Mixed Models
}

\author{
By
}

\section{JANARDHAN BARAL}

\author{
A thesis submitted to \\ the Faculty of Graduate Studies and Research \\ in partial fulfilment of \\ the requirements for the degree of \\ Master of Science
}

School of Mathematics and Statistics

Ottawa-Carleton Institute of Mathematics and Statistics

Carleton University

Ottawa, Ontario, Canada

August 2006

๑) Copyright by Janardhan Baral, 2006 


$\begin{array}{ll}\begin{array}{l}\text { Library and } \\ \text { Archives Canada }\end{array} & \begin{array}{l}\text { Bibliothèque et } \\ \text { Archives Canada }\end{array} \\ \begin{array}{l}\text { Published Heritage } \\ \text { Branch }\end{array} & \begin{array}{l}\text { Direction du } \\ \text { Patrimoine de l'édition }\end{array} \\ \begin{array}{l}\text { 395 Wellington Street } \\ \text { Ottawa ON K1A 0N4 }\end{array} & \begin{array}{l}\text { 395, rue Wellington } \\ \text { Ottana ON K1A ON4 } \\ \text { Canada Oa }\end{array}\end{array}$

Your file Votre référence ISBN: 978-0-494-26224-5 Our file Notre référence ISBN: 978-0-494-26224-5

NOTICE:

The author has granted a nonexclusive license allowing Library and Archives Canada to reproduce, publish, archive, preserve, conserve, communicate to the public by telecommunication or on the Internet, loan, distribute and sell theses worldwide, for commercial or noncommercial purposes, in microform, paper, electronic and/or any other formats.

The author retains copyright ownership and moral rights in this thesis. Neither the thesis nor substantial extracts from it may be printed or otherwise reproduced without the author's permission.
AVIS:

L'auteur a accordé une licence non exclusive permettant à la Bibliothèque et Archives Canada de reproduire, publier, archiver, sauvegarder, conserver, transmettre au public par télécommunication ou par l'Internet, prêter, distribuer et vendre des thèses partout dans le monde, à des fins commerciales ou autres, sur support microforme, papier, électronique et/ou autres formats.

L'auteur conserve la propriété du droit d'auteur et des droits moraux qui protège cette thèse. $\mathrm{Ni}$ la thèse ni des extraits substantiels de celle-ci ne doivent être imprimés ou autrement reproduits sans son autorisation.
In compliance with the Canadian

Privacy Act some supporting forms may have been removed from this thesis.

While these forms may be included in the document page count, their removal does not represent any loss of content from the thesis.
Conformément à la loi canadienne sur la protection de la vie privée, quelques formulaires secondaires ont été enlevés de cette thèse.

Bien que ces formulaires aient inclus dans la pagination, il n'y aura aucun contenu manquant. 


\section{Abstract}

In this thesis, we investigate the impact of model violations on the estimates of regression coefficients in a generalized linear mixed model (GLMM). The penalized quasi-likelihood (PQL), which is an approximate method of inference in GLMMs, converges to a well defined limit. Recent developments of statistical techniques, which include Gaussian quadrature, Laplace expansions, Markov Chain Monte Carlo, provide attractive alternatives to the PQL for approximate likelihood inference in GLMMs. These methods are usually based on the assumption that the distribution of the vector of random effects in GLMMs is multivariate normal with a certain mean vector and a covariance matrix. Here we study the bias of the regression estimates that result from incorrect assumptions regarding the random effects. We ran a number of simulations to compare the impact of model violations on the parameter estimates obtained from two methods, which include the $P Q L$ and the ML methods. The PQL method appears to provide more robust estimates for random effects model misspecifiction as compared to the ML method. 


\section{Acknowledgements}

I would like to thank my supervisor Dr. Sanjoy Sinha for his tireless efforts and patience in making this thesis become a reality. I gratefully acknowledge the partial financial support for this research provided from my supervisor's research grant. I also thank the School of Mathematics and Statistics at Carleton University for providing me the opportunity to pursue graduate study in Statistics. It is certainly a privilege to thank Valerie Daley, the Graduate Administrator of the School, and Dr. Shirley Mills for their valuable suggestions and advice for my study at Carleton. Many thank to my wife Pratima Das, my lovely daughters Chelsia and Shinthea and my friend Suresh Datta for their valuable support. 


\section{Contents}

$\begin{array}{ll}\text { Abstract } & \text { ii }\end{array}$

Acknowledgements $\quad$ iii

Contents iv

List of Tables vii

List of abbreviation $\quad \mathbf{x}$

1 Introduction 1

2 Generalized Linear Models $\quad 5$

2.1 Introduction 5

2.2 Components 6

$\begin{array}{ll}2.3 \text { Likelihood } & 7\end{array}$

2.4 Link Functions $\quad 8$

2.5 Fitting GLMs 9

2.6 Quasi-likelihood 11

2.6.1 Construction 12

2.6.2 Quasi-deviance 13

2.6.3 Estimation 13

3 Generalized Linear Mixed Models 17

$\begin{array}{ll}3.1 \text { Introduction } & 17\end{array}$ 
3.3 Consequences of Having Random Effects

3.3.1 Marginal VS Conditional Distribution

3.3.2 Mean of $y$

3.3.3 Variances

3.3.4 Covariance and Correlation

$\begin{array}{ll}\text { 3.4 Likelihood Estimation } & 22\end{array}$

3.4.1 Fixed effects Parameters 25

3.4.2 Random Effects Parameters 26

$\begin{array}{ll}3.5 \text { Penalized Quasi-likelihood } & 26\end{array}$

3.5.1 Likelihood Estimation 28

3.5.2 Variance Component Estimation 31

3.6 Computing ML estimates 32

3.6.1 Numerical quadrature $\quad 32$

3.6.2 Gauss-Hermite quadrature 34

3.6.3 Likelihood calculations 34

3.6.4 Monte Carlo EM 36

3.6.5 Monte Carlo Newton-Raphson $\quad 37$

3.6.6 Simulated maximum likelihood 38

4 Missspecified Generalized Linear Mixed Model $\quad 40$

4.1 Introduction 40

4.2 Asymptotic Bias of ML estimates for Misspecified Models 42

4.3 Illustrative example 43 
4.3.1 Non-Gaussian random effects 44

4.3.2 Heterogeneity depends on a cluster-level covariates 44

4.3.3 Random effects $\quad 45$

4.3.4 Autoregressive random effects 46

$5 \quad$ Simulation Study $\quad 47$

$\begin{array}{lll}5.1 & \text { Non-Gaussian random effects } & 47\end{array}$

5.2 Heterogeneity dependent on a binary-cluster level covariates 51

5.3 Random intercepts and random slopes are mutually independent 57

5.4 Autoregressive random effects 62

Conclusion 66

Appendix: R codes used for Simulation Study

A1: $\mathrm{R}$ codes when Random Intercepts are Non-Gaussian 


\section{List of Tables}

1.1 Simulated biases for ML and PQL estimates under misspecified Gaussian random effects for GLMM's where the random effects have a gamma distribution, $u_{i j}=u_{i, 0}=\sigma\left(a_{i}-\lambda\right) / \sqrt{\lambda}$ for $a_{i} \sim \operatorname{gamma}(\lambda, 1), \mathbf{k}=(40$, 200 ), simulation $=500$ and $n=5$.

1.2 Simulated root mean squared errors for ML and PQL estimates under misspecified Gaussian random effects for GLMM's where the random effects have a gamma distribution, $u_{i j}=u_{i, 0}=\sigma\left(a_{i}-\lambda\right) / \sqrt{\lambda}$ for $a_{i} \sim \operatorname{gamma}(\lambda, 1), \mathrm{k}=(40,200)$, simulation $=500$ and $\mathrm{n}=5$.

2.1 Simulated biases for ML and PQL estimates under misspecified Gaussian random effects for GLMM's where the variance of the random effects depends on a between-cluster covariate: $\left[u_{i, 0} \mid X_{i j, 1}=0\right] \sim N\left(0, \sigma_{0}{ }^{2}\right)$ and $\left[u_{i, 0} \mid X_{i j, 1}=1\right] \sim N\left(0, \sigma_{1}{ }^{2}\right), \mathrm{k}=40$, simulation $=500$ and $\mathrm{n}=5$.

2.2 Simulated biases for ML and PQL estimates under misspecified Gaussian random effects for GLMM's where the variance of the random effects depends on a between-cluster covariate: $\left[u_{i, 0} \mid X_{i j, 1}=0\right] \sim N\left(0, \sigma_{0}{ }^{2}\right)$ and $\left[u_{i, 0} \mid X_{i j, 1}=1\right] \sim N\left(0, \sigma_{1}^{2}\right), \mathrm{k}=200$, simulation $=500$ and $\mathrm{n}=5$. 
2.3 Root mean squared error for ML and PQL estimates under misspecified Gaussian random effects for GLMM's where the variance of the random effects depends on a between-cluster covariate $\left[u_{i, 0} \mid X_{i j, 1}=0\right] \sim N\left(0, \sigma_{0}{ }^{2}\right)$ and $\left[u_{i, 0} \mid X_{i j, 1}=1\right] \sim N\left(0, \sigma_{1}^{2}\right), \mathbf{k}=40$, simulation $=500$ and $\mathbf{n}=5$.

2.4 Root mean squared error for $\mathrm{ML}$ and PQL estimates under misspecified Gaussian random effects for GLMM's where the variance of the random effects depends on a between-cluster covariate: $\left[u_{i, 0} \mid X_{i j, 1}=0\right] \sim N\left(0, \sigma_{0}{ }^{2}\right)$ and $\left[u_{i, 0} \mid X_{i j, 1}=1\right] \sim N\left(0, \sigma_{1}^{2}\right), \mathrm{k}=200$, simulation $=500$ and $\mathrm{n}=5$.

3.1 Simulated biases for ML and PQL estimates under misspecified Gaussian random effects for GLMM's, when there are random intercepts and random slopes are mutually independent: $u_{i j}=u_{i, 0}+u_{i, 1} x_{j}$ with $u_{i, 0} \sim N\left(0, \sigma_{0}^{2}\right)$ and $u_{i, 1} \sim N\left(0, \sigma_{1}^{2}\right), \mathrm{k}=40$, simulation $=500$ and $\mathrm{n}=5$.

3.2 Simulated biases for ML and PQL estimates under misspecified Gaussian random effects for GLMM's, when there are random intercepts and random slopes are mutually independent: with $u_{i, 0} \sim N\left(0, \sigma_{0}^{2}\right)$ and $u_{i, 1} \sim N\left(0, \sigma_{1}^{2}\right)$, $\mathbf{k}=200$, simulation $=500$ and $n=5$.

3.3 Root mean squared errors for $\mathrm{ML}$ and $\mathrm{PQL}$ estimates under misspecified Gaussian random effects for GLMM's, when there are random intercepts and random slopes are mutually independent: $u_{i j}=u_{i, 0}+u_{i, 1} x_{j}$ with $u_{i, 0} \sim N\left(0, \sigma_{0}^{2}\right)$ and $u_{i, 1} \sim N\left(0, \sigma_{1}^{2}\right), \mathrm{k}=40$, simulation $=500$ and $\mathrm{n}=5$.

3.4 Root mean squared errors for ML and PQL estimates under misspecified Gaussian random effects for GLMM's, when there are random intercepts and random slopes are mutually independent: $u_{i j}=u_{i, 0}+u_{i, 1} x_{j}$ and 
with $u_{i, 0} \sim N\left(0, \sigma_{0}^{2}\right)$ and $u_{i, 1} \sim N\left(0, \sigma_{1}^{2}\right), \mathrm{k}=200$, simulation $=500$ and $\mathrm{n}=5$.

4.1 Simulated biases for ML and PQL estimates under misspecified Gaussian random effects for GLMM's, but the random effects are autocorrelated: $\operatorname{cov}\left(u_{i j}, u_{i k}\right)=\sigma^{2} \rho^{|j-k|}$ with $\mathrm{k}=(40,200)$, simulation $=500$ and $\mathrm{n}=5$.

4.2 Root mean squared error for ML and PQL estimates under misspecified Gaussian random effects for GLMM's, but the random effects are autocorrelated: $\operatorname{cov}\left(u_{i j}, u_{i k}\right)=\sigma^{2} \rho^{|j-k|}$ with $\mathrm{k}=(40,200)$, simulation=500 and $n=5$. 


\section{List of abbreviation}

GLM=Generalized linear model

GLMM $=$ Generalized linear mixed model

PQL=Penalized quasi-likelihood

MLE=Maximum likelihood estimators

MCMC $=$ Markov chain Monte Carlo

MCNR= Monte Carlo Newton-Raphson

$\mathrm{MSE}=$ Mean squared error

QMLE $=$ Quasi maximum likelihood estimators

$\mathrm{SML}=$ Simulated maximum likelihood 


\section{Chapter 1}

\section{Introduction}

Generalized linear mixed models (GLMM's) are widely used in the analysis of clustered data, including longitudinal data or repeated measurements (Breslow and Clayton 1993). GLMM's are useful for accommodating the overdispersion often observed among nonnormally distributed responses and for modeling the dependence among responses inherent in longitudinal or repeated measures data by incorporating random effects (Stiratelli, Laird, and Ware 1984; Zeger, Liang, and Albert 1988). It is usually assumed that the random effects have a multivariate normal distribution whose variance components are to be estimated from the data. A full maximum likelihood.(ML) analysis based on the joint marginal likelihood of the responses can be used for estimating both fixed and random effects parameters in GLMM's, which requires numerical integration techniques for calculating the log-likelihood, score equations, and information matrix. However, its use in GLMM's is limited to relatively simple models, and it is found intractable for more complicated problems involving irreducibly high-dimensional integrals. 
To avoid such computational problems, a number of Bayesian approaches have been suggested that generate repeated samples from the posterior distributions of the random effects using Gibbs Sampling techniques (Besag, York, and Mollie 1991; Zeger and Karim 1991). The approach of generalized estimating equations (GEE's) (Diggle, Liang, and Zeger 1994) is useful for the analysis of longitudinal data, but it suffers from a lack of efficiency (Crowder 1995; Fitzmaurice 1995; Sutradhar and Das 1999). In a recent work, Vonesh, Wang, Nie, and Majumder (2002) proposed conditionally second order generalized estimating equations (CGEE2's) for estimating the parameters in GLMM's. Under appropriate regularity conditions, the CGEE2 estimator is shown to be consistent and asymptotically efficient. McCulloch (1997) developed a Monte Carlo NewtonRaphson (MCNR) algorithm for approximating the ML estimates in GLMM's. The MCNR estimates were compared to the exact ML estimates for simple models, and it was found that MCNR inherits the properties of the exact ML estimates. Recently, Sutradhar and Sinha (2002) developed a Psedo-likelihood approach for estimating the variance components of a binary longitudinal mixed model. This approach is developed under the assumption that the variance components of the random effects are small in magnitude.

However, the sensitivity of the estimated regression coefficients to random effects assumptions in mixed models has only been explored to a limited degree. Neuhaus et al. (1992) investigate the effects of mixture distribution misspecification when fitting mixed-effects logistic models. They concluded that although regression estimates are asymptotically biased, the magnitude of bias is typically small. Liang and Hanfelt (1994) considered likelihood inference using the beta binomial model and showed that ignoring variation in the withincluster correlation as a function of covariates can result in severe bias for regression estimates. Molenberghs et al. (1998) studied the performance of test statistics based on misspecified models. Ten Have et al. (1999) studied binary data with multiple levels of clustering and showed that fitting an incomplete multilevel structure can lead to bias in the estimates. Tan et al. (1999) showed that using generalized estimating equations (Liang and Zeger, 1986) for an underlying latent variable model does not lead to biased estimation or inference. 
Palta et al. (1997) presented a general study of omitted confounders and other forms of misspecification when models are fitted using generalized estimating equations. Even though the PQL approach is widely used in many different applications, it is well-known that estimating the variance components is quite challenging due to their non-observability. Accordingly, several different types of likelihood functions of the variance components have been suggested including a maximum adjusted profile h-likelihood estimator (MAPHLE) by Lee and Nelder (1996).

For the linear mixed model, the selection of proper a random effects structure is necessary for efficient estimation and for proper model-based standard error estimates. Misspecification of the covariance of does not affect the consistency of the maximum likelihood regression estimator. For generalized linear mixed models, the use of non-linear link functions means that the proper selection of the random effects structure may be required for both valid point estimates and for correct standard error. Ten Have et al. (1999), Heagerty (1999) and Heagerty and Zeger (2000) illustrate that generalized linear mixed model estimates can be sensitive to the random effect assumptions.

In this thesis, we study the impact of misspecification of the random effects assumptions on some commonly used methods for fitting generalized linear mixed models. A series of simulations were carried out in which we investigate the bias and root mean squared errors of the ML and PQL estimates of regression coefficients in GLMM's with incorrectly specified random effects distributions.

In chapter 2, we introduce the generalized linear models (GLMs) and review some commonly used methods for inference in the GLM's. In chapter 3, we describe the generalized linear mixed models (GLMMs) as an extension of the GLM's for analyzing clustered data including longitudinal data or repeated measurements. We also briefly review the maximum likelihood and the penalized quasi-likelihood methods for inference in the GLMM's. 
In chapter 4, we discuss some illustrative examples where the relevant regression model is correctly specified, but the distributional assumptions for the random effects are misspecified. In chapter 5 , we ran a series of simulations to investigate the biases and root mean squared errors of parameter estimates for the misspecified generalized linear mixed models. We compare the classical maximum likelihood estimates to the penalized quasi-likelihood estimates for fitting the mixed models with incorrect random effects distributions. Finally, concluding remarks are made in Chapter 6. 


\section{Chapter 2}

\section{Generalized Linear Models}

\subsection{Introduction}

The development of the General Linear Regression model for Normal data was the great development of Statistics in the early 20th century. In this precomputer age there was much effort devoted to experimental designs that would, not only be statistically efficient, but be easily analyzed by hand computation. Fisher and Yates were two of the pioneers of this work, both working at the Rothamsted agricultural research station. With the advent of computers the effort of computation was removed and statistical research moved on to the extension of these methods to a wider class of statistical problem, in particular non-Normal data. Much of the impetus come from biostatistics in which it was common that observations were binary, that is there were only two possible outcomes e.g. dead or alive. Numerical procedures were devised for each situation in isolation and computer programs for model fitting were specific to that situation. A breakthrough came with the work of Nelder and Wedderburn (also working at Rothamsted) who devised a wide class 
of models that could all be fitted by a common numerical algorithm, the Generalized Linear Model (1972).

Hypothesis tests applied to the Generalized Linear Model do not require normality of the response variable, nor do they require homogeneity of variances. Hence, Generalized Linear Models can be used when response variables follow distributions other than the Normal distribution, and when variances are not constant. For example, count data would be appropriately analyzed as a Poisson random variable within the context of the Generalized Linear Model.

\subsection{The Components of a Generalized Linear model}

Generalized linear models are an extension of classical linear models. The classical linear model can be summarized as follows:

The components of $y$ are independent normal variables with constant variance $\sigma^{2}$ and response mean vector

$$
E(y)=\mu
$$

where

$$
\mu=\left(\mu_{1}, \mu_{2}, \cdots, \mu_{n}\right)^{\prime} \text { with } \mu_{i}=E\left(y_{i}\right)=\sum_{j=1}^{p} x_{i j} \beta_{j} .
$$

The above model has the following three specific components:

1. The "random component": The random components of $y$ are independently normally distributed with $E(y)=\mu$ and constant variance $\sigma^{2}$;

2. The "systematic component": covariates $x_{1}, x_{2}, \ldots . . x_{p}$ produce a "linear predictor" $\eta$ given by $\eta=\sum_{j=1}^{p} x_{j} \beta_{j}$ and

3. The "link" between random and systematic components, $\mu=\eta$. 
Now if we write $\eta_{i}=g\left(\mu_{i}\right)$, then $g$ is called the "link function".

In this formulation, classical linear models have a normal distribution in component 1 and the identity function for the link in component 3. Generalized linear models allow two extensions; first, the distribution in component 1 comes from an exponential family other than the normal, and second, the link function in component 3 may become any monotonic differentiable function.

\subsection{Likelihood Functions for GLMs}

We assume that each component of $y$ has a distribution in the exponential family, taking the form:

$$
f(y, \theta, \phi)=\exp \left\{\left(\frac{y \theta-b(\theta)}{a(\phi)}\right)+c(y, \phi)\right\}
$$

where, $\phi$ is the scale parameter and $\theta$ is called the natural location for some functions $a, b$ and $c$. For members of the exponential family, if $\phi$ is known, this is an exponential family model with conditional parameter $\theta$. We write,

$$
l(\theta, \phi ; y)=\log f(y ; \theta, \phi)=\left\{\left(\frac{y \theta-b(\theta)}{a(\phi)}\right)+c(y, \phi)\right\}
$$

for the $\log$ likelihood function considered as a function of $\theta$ and $\phi, \mathrm{y}$ being given. The mean and variance can be easily derived from the well- known relations

$$
E\left(\frac{\partial l}{\partial \theta}\right)=0
$$

and

$$
E\left(\frac{\partial^{2} l}{\partial \theta^{2}}\right)+E\left(\frac{\partial l}{\partial \theta}\right)^{2}=0
$$


From $(2 \cdot 2)$, we have

$$
\frac{\partial l}{\partial \theta}=\left\{\frac{y-b^{\prime}(\theta)}{a(\phi)}\right\}
$$

and

$$
\frac{\partial^{2} l}{\partial \theta^{2}}=\frac{-b^{\prime \prime}(\theta)}{a(\phi)}
$$

From $(2 \cdot 3)$ and $(2 \cdot 6)$, we have

$$
E\left(\frac{\partial l}{\partial \theta}\right)=\left\{\frac{\mu-b^{\prime}(\theta)}{a(\phi)}\right\}
$$

so that

$$
E(y)=\mu=b^{\prime}(\theta)
$$

Similarly, from (2.4) and $(2 \cdot 7)$,

$$
0 \equiv-\frac{b^{\prime \prime}(\theta)}{a(\phi)}+\frac{\nu(y)}{a^{2}(\phi)}
$$

so that

$$
\operatorname{var}(y)=b^{n}(\theta) a(\phi)
$$

Thus the variance of $\mathrm{y}$ is the product of two functions; one, $b^{\prime \prime}(\theta)$, depends on the canonical parameter (and hence on the mean) only and will be called the variance function, while the other is independent of $\theta$ and depends only on $\phi$.

\subsection{Link functions}

A link function relates the linear predictor $\eta_{i}=x_{i}^{\prime} \beta$ to the mean response $\mu_{i}$ :

$$
\begin{aligned}
& E\left[y_{i}\right]=\mu_{i} \\
& g\left(\mu_{i}\right)=\eta_{i}=x_{i}^{\prime} \beta .
\end{aligned}
$$


Each distribution in the exponential family has a link function for which there exists a sufficient statistic. A canonical link occurs when $\theta=\eta$, where $\theta$ is the canonical parameter in the exponential family.

In classical linear models, the mean and the linear predictor are identical, and the identity link is plausible in that both $\eta$ and $\mu$ can take any value on the real line. However, when we are dealing with counts and the distribution is Poisson, we must have $\mu>0$, so that the identity link is less attractive, in part because $\eta$ may be negative while $\mu$ not be. Models for counts based on independence in cross-classified data lead naturally to multiplicative effects, and this is expressed by the $\log \operatorname{link}, \eta=\log \mu$, with its inverse $\mu=e^{\eta}$. Now additive effects contributing to $\eta$ become multiplicative effects contributing to $\mu$, and $\mu$ is necessarily positive.

For the binomial distribution, we have $0<\mu<1$ and a link should satisfy the condition that it maps the interval $(0,1)$ on to the whole real line. Now we can consider three-principal functions, namely:

$\begin{array}{ccc}\text { 1. logit: } & & \eta=\log \left(\frac{\mu}{1-\mu}\right) \\ \ldots & \ldots(2 \cdot 12) & =-1 / \text { । }\end{array}$

where $\Phi(\cdot)$ is the Normal cumulative distribution function;

3. complementary log-log: $\quad \eta=\log \{-\log (1-\mu)\}$. In;

3. complementary log-log: $\quad \eta=\log \{-\log (1-\mu)\}$.

\subsection{Fitting GLMs using the ML method}

The log likelihood for a single observation, in canonical form, is given by,

$$
l_{i}=\frac{y_{i} \theta_{i}-b\left(\theta_{i}\right)}{a_{i}(\phi)}+c\left(y_{i}, \phi\right)
$$


Now differentiating $(2 \cdot 14)$ with respect to $\beta_{j}$,

$$
\begin{aligned}
\frac{\partial l_{i}}{\partial \beta_{j}} & =\frac{\partial l_{i}}{\partial \theta_{i}} \cdot \frac{\partial \theta_{i}}{\partial \mu_{i}} \cdot \frac{\partial \mu_{i}}{\partial \eta_{i}} \cdot \frac{\partial \eta_{i}}{\partial \beta_{j}} \\
& =\frac{y_{i}-b^{\prime}\left(\theta_{i}\right)}{a_{i}(\phi)} \cdot \frac{1}{b^{\prime \prime}\left(\theta_{i}\right)} \cdot \frac{\partial \mu_{i}}{\partial \eta_{i}} \cdot x_{j i} \\
& =\left(y_{i}-\mu_{i}\right) \cdot \frac{1}{a_{i}(\theta) \cdot b^{\prime \prime}\left(\theta_{i}\right)} \cdot \frac{\partial \mu_{i}}{\partial \eta_{i}} \cdot x_{j i} \\
& =\left(y_{i}-\mu_{i}\right) \cdot \frac{1}{v\left(y_{i}\right)} \cdot\left(\frac{\partial \mu_{i}}{\partial \eta_{i}}\right)^{2} \cdot\left(\frac{\partial \eta_{i}}{\partial \mu_{i}}\right) \cdot x_{j i}
\end{aligned}
$$

For a set of $\mathrm{n}$ observations $y_{1}, y_{2}, \ldots, y_{n}(2 \cdot 15)$ can be written in matrix form as

$$
\frac{\partial l}{\partial \beta_{j}}=(y-\mu) \cdot w \cdot d \cdot x_{j}
$$

where

$$
w=\frac{1}{v\left(y_{i}\right)} \cdot\left(\frac{\partial \mu_{i}}{\partial \eta_{i}}\right)^{2} \text { is called weight matrix and the dispersion }
$$

parameter $d=\left(\frac{\partial \eta_{i}}{\partial \mu_{i}}\right)$

By Taylor series expansion, we can write

$$
\left.0 \equiv \frac{\partial l}{\partial \beta} \cong \frac{\partial l}{\partial \beta}\right|_{\beta_{0}}+\left.\frac{\partial^{2} l}{\partial \beta \partial \beta^{\prime}}\right|_{\beta_{0}}\left(\beta-\beta_{0}\right)
$$

where

$$
\left.\frac{\partial^{2} l}{\partial \beta \partial \beta^{\prime}}\right|_{\beta_{0}}=I_{0}\left(\beta_{0}\right) \text { is called Fisher observed information. In matrix }
$$

notation, $(2 \cdot 15)$ can be written as 


$$
\left.\frac{\partial l}{\partial \beta}\right|_{\beta_{0}}=X^{\prime} W_{0} D_{0}\left(y-\mu_{0}\right)
$$

Fishers scoring technique replaces $I_{0}\left(\beta_{0}\right)$ in $(2 \cdot 16)$ by $I_{E}\left(\beta_{0}\right)$ where

$$
I_{E}\left(\beta_{0}\right)=-\left.E\left(\frac{\partial^{2} l}{\partial \beta \partial \beta^{\prime}}\right)\right|_{\beta_{0}} .
$$

From $(2 \cdot 15)$

$$
\frac{\partial l}{\partial \beta_{j}}=\sum w(y-\mu) \frac{\partial \eta}{\partial \mu} x_{j}
$$

Now differentiating $(2 \cdot 18)$ with respect to $\beta_{k}$, we get

$$
\frac{\partial^{2} l}{\partial \beta_{j} \partial \beta_{k}}=\sum\left[(y-\mu) \cdot \frac{\partial}{\partial \beta_{k}}\left(w \cdot \frac{\partial \eta}{\partial \beta_{k}} \cdot x_{j}\right)+\left(w \cdot \frac{\partial \eta}{\partial \mu} x_{j}\right) \cdot \frac{\partial}{\partial \beta_{k}}(y-\mu)\right]
$$

so that

$$
E\left(\frac{\partial^{2} l}{\partial \beta_{j} \partial \beta_{k}}\right)=-\sum w \cdot \frac{\partial \eta}{\partial \mu} \cdot x_{j} \cdot \frac{\partial \mu}{\partial \beta_{k}}
$$

In matrix notation:

$$
I_{E}\left(\beta_{0}\right)=-\left.E\left(\frac{\partial^{2} l}{\partial \beta \partial \beta^{\prime}}\right)\right|_{\beta_{0}}=X^{\prime} W_{0} X
$$

Hence from $(2 \cdot 16)$, we find

$$
\begin{aligned}
& \left.\frac{\partial l}{\partial \beta}\right|_{\beta_{0}} \approx I_{E}\left(\beta_{0}\right)\left(\beta-\beta_{0}\right) \\
& \beta \cong \beta_{0}+\left[I_{E}\left(\beta_{0}\right)\right]^{-1}\left(\left.\frac{\partial l}{\partial \beta}\right|_{\beta_{0}}\right)
\end{aligned}
$$




$$
\begin{aligned}
& =\beta_{0}+\left(X^{\prime} W_{0} X\right)^{-1} X^{\prime} W_{0} D_{0}\left(y-\mu_{0}\right) \\
& =\left(X^{\prime} W_{0} X\right)^{-1} X^{\prime} W_{0}\left\{X \beta_{0}+D_{0}\left(y-\mu_{0}\right)\right\} \\
& =\left(X^{\prime} W_{0} X\right)^{-1} X^{\prime} W_{0} Z_{0}
\end{aligned}
$$

where

$$
Z_{0}=x \beta_{0}+D_{0}\left(y-\mu_{0}\right) \text { is called a pseudo vector. }
$$

In particular

$$
\begin{aligned}
& z_{i 0}=x_{i} \beta_{0}+\left.\frac{\partial \eta_{i}}{\partial \mu_{i}}\right|_{\mu_{i 0}}\left(y_{i}-\mu_{i 0}\right) \\
& =\eta_{i}+\left.\frac{\partial \eta_{i}}{\partial \mu_{i}}\right|_{\mu_{i 0}}\left(y_{i}-\mu_{i 0}\right) \\
& =g\left(\mu_{i 0}\right)+\left.\frac{\partial \eta_{i}}{\partial \mu_{i}}\right|_{\mu_{i 0}}\left(y_{i}-\mu_{i 0}\right)
\end{aligned}
$$

and

$$
v\left(z_{i 0}\right)=\eta_{i}+\left(\left.\frac{\partial \eta_{i}}{\partial \mu_{i}}\right|_{\mu_{i 0}}\right)^{2} \cdot v\left(y_{i}\right)
$$

Thus, we can use weighted regression of $z_{0}$ on $x$ with weights $w_{0}$. This method is referred to as the Iteratively Reweighted Least Square (IRWLS) method.

\subsection{Quasi -likelihood}

In-many problems of statistical estimation, we know some-detail of the distribution governing the data, but may be unwilling to specify it exactly. This precludes the use of maximum likelihood, which required exact specification of the distribution in order to construct the likelihood. The idea of quasilikelihood, developed by Wedderburn (1974), addresses this concern. This is a method of estimation that requires only a model for the mean of the data and the relationship between the mean and the variance, yet in many cases retain full or nearly full efficiency compared to maximum likelihood. 
We can use estimating equations very similar to the likelihood equations for the exponential family, without actually assuming the underlying distributions.

For the exponential family of $(2 \cdot 1)$

$$
l=\sum_{i=1}^{n}\left\{\frac{y_{i} \theta_{i}-b\left(\theta_{i}\right)}{a_{i}(\phi)}+c\left(y_{i} ; \theta\right)\right\}
$$

where

$$
E(y)=\mu=b^{\prime}(\theta)
$$

and

$$
v\left(y_{i}\right)=a_{i}(\phi) \cdot b^{\prime \prime}\left(\theta_{i}\right)=a_{i}(\phi) \cdot v\left(\mu_{i}\right)
$$

Suppose, $v\left(y_{i}\right)=\sigma^{2} v\left(\mu_{i}\right)$ which allows over or under dispersion, where $\sigma^{2}$ may be unknown and $v\left(\mu_{i}\right)$ is a known factor.

\subsubsection{Construction}

Consider a single observation $y$ and assume $y \sim \cdot\left(\mu, \sigma^{2} v(\mu)\right)$ that is $E(y)=\mu$ and $v(y)=\sigma^{2} v(\mu)$ where $v(\mu)$ is a variance function. Let,

$$
\begin{aligned}
& u=u(\mu ; y)=\frac{y-\mu}{\sigma^{2} v(\mu)} \\
& \frac{\partial u}{\partial \mu}=\frac{\partial}{\partial \mu}\left(\frac{y-\mu}{\sigma^{2} v(\mu)}\right) \\
& =\frac{1}{\sigma^{2}}\left(\frac{-v(\mu)-(y-\mu) v^{\prime}(\mu)}{[v(\mu)]^{2}}\right)
\end{aligned}
$$

Therefore,

$$
E\left(\frac{\partial u}{\partial \mu}\right)=-\frac{1}{\sigma^{2} v(\mu)}
$$


and it has the following properties:

$$
\begin{gathered}
E(u)=0 \\
v(u)=\frac{1}{\sigma^{2} v(\mu)} \\
\text { and }-E\left(\frac{\partial u}{\partial \mu}\right)=\frac{1}{\sigma^{2} v(\mu)} .
\end{gathered}
$$

Therefore, $u$ behaves like the score function. Most first order asymptotic theory connected with likelihood functions is founded on the above three properties. So it is not surprising that to some extent, the integral

$$
Q(\mu ; y)=\int_{y}^{\mu} \frac{y-t}{\sigma^{2} v(t)} d t
$$

if it exists, should behave like a log-likelihood function for $\mu$ under some regularity conditions. We refer to $Q(\mu ; y)$ as the quasi-likelihood, or more correctly, as the $\log$ quasi-likelihood for $\mu$ based on data $y$. Since the components of $y$ are independent by assumption, the quasi-likelihood for the complete data is the sum of the individual contributions $Q(\mu ; y)=-\sum Q_{i}\left(\mu_{i} ; y_{i}\right)$.

\subsubsection{Quasi-deviance}

A corresponding goodness-of-fit criterion, the quasi-deviance, is defined analogously to the deviance in the generalized linear model and is given by

$$
D(y ; \mu)=-2 \sigma^{2} Q(\mu ; y)=2 \int_{\mu}^{y} \frac{y-\mu}{v(t)} d t
$$


which is evidently strictly positive except at $y=\mu$. For $\mathrm{n}$ observations $y_{1}, y_{2}, y_{3}, \ldots . . ., y_{n}$, the total deviance $D(y ; \mu)=\sum_{i} D_{i}\left(y_{i} ; \mu_{i}\right)$, obtained by adding over the components, is a computable function depending on $y$ and $\mu$ alone and it does not depend on $\sigma^{2}$. A factor 2 is added because differences in the deviances between nested models will then follow an asymptotic $\chi^{2}$ distribution. Maximizing the quasi-likelihood is equivalent to minimizing the quasi-deviance.

\subsubsection{Parameter Estimation}

We can use Newton-Raphson with Fisher scoring technique (i.e., replace "observation information" by "expected information"). We have

$$
\begin{aligned}
& \frac{\partial Q}{\partial \beta}=\sum_{i} \frac{\partial Q_{i}}{\partial \beta} \\
& =\sum_{i} \frac{\partial Q_{i}}{\partial \mu_{i}} \frac{\partial \mu_{i}}{\partial \eta_{i}} \frac{\partial \eta_{i}}{\partial \beta} \\
& =\sum_{i} u\left(\mu_{i} ; y_{i}\right) \frac{\partial \mu_{i}}{\partial \eta_{i}} x_{i} \\
& =\sum_{i} \frac{y_{i}-\mu_{i}}{\sigma^{2} v\left(\mu_{i}\right)} \cdot \frac{\partial \mu_{i}}{\partial \eta_{i}} \cdot x_{i}
\end{aligned}
$$

In matrix form,

$$
\frac{\partial Q}{\partial \beta}=\frac{D^{\prime} V^{-1}(y-\mu)}{\sigma^{2}} \text { is called-the quasi-score function, }
$$

where

$$
V^{-1}=1 / v\left(\mu_{i}\right) \text { and } D=\frac{\partial \mu}{\sigma \eta_{i}} x_{i}
$$

Also

$$
\frac{\partial^{2} Q}{\partial \beta \partial \beta^{\prime}}=\frac{1}{\sigma^{2}}\left[(y-\mu) \frac{\partial}{\partial \beta}\left(D^{\prime} V^{-1}\right)+D^{\prime} V^{-1} \frac{\partial}{\partial \beta}(y-\mu)\right]
$$


and

$$
-E\left(\frac{\partial^{2} Q}{\partial \beta \partial \beta^{\prime}}\right)=\frac{D^{\prime} V^{-1} D}{\sigma^{2}}=I_{E}(\beta)
$$

where $I_{E}(\beta)$ is the Fisher Information matrix. Now, by Taylor series expansion,

we get

$$
0 \equiv \frac{\partial Q}{\partial \beta}=\left.\frac{\partial Q}{\partial \beta}\right|_{\beta_{0}}+\left.\frac{\partial^{2} Q}{\partial \beta \partial \beta^{\prime}}\right|_{\beta_{0}}\left(\beta-\beta_{0}\right)
$$

Using Fisher scoring technique, we have

$$
\begin{aligned}
& \left.\left.\frac{\partial Q}{\partial \beta}\right|_{\beta_{0}} \cong E\left(-\frac{\partial^{2} Q}{\partial \beta \partial \beta^{\prime}}\right)\right|_{\beta_{0}}\left(\beta-\beta_{0}\right) \\
& \Rightarrow \frac{D_{0}^{\prime} V_{0}^{-1}\left(y-\mu_{0}\right)}{\sigma^{2}} \cong\left(\frac{D_{0}^{\prime} V_{0}^{-1} D_{0}}{\sigma^{2}}\right)\left(\beta-\beta_{0}\right) \\
& \Rightarrow \beta \cong \beta_{0}+\left(D_{0}^{\prime} V_{0}^{-1} D_{0}\right)^{-1} D_{0}^{\prime} V_{0}^{-1}\left(y-\mu_{0}\right)
\end{aligned}
$$

We can solve for $\beta$ iteratively from the equation

$$
\beta_{(k+1)}=\beta_{(k)}+\left(D_{(k)}^{\prime} V_{(k)}^{-1} D_{(k)}\right)^{-1} D_{(k)}^{\prime} V_{(k)}^{-1}\left(y-\mu_{(k)}\right)
$$

For $\mathrm{k}=0, \ldots 1,2 \ldots$ Note that the estimate of $\beta$ does not depend on the value $\sigma^{2}$. Under suitable regularity conditions, the quasi-likelihood estimate $\hat{\beta}$ follows an asymptotic normal distribution with mean vector $\beta$ and covariance matrix $\sigma^{2}\left(D^{\prime} V^{-1} D\right)^{-1}$ 


\section{Chapter 3}

\section{Generalized Linear Mixed Models}

\subsection{Introduction}

Generalized linear mixed models are a natural extension of both linear mixed models and generalized linear models. They are useful for the accommodation of nonnormally distributed responses and specification of a possibly nonlinear link between the mean response and the predictors, and for calculating overdispersion and correlation by incorporating random effects. Generalized linear mixed models are particularly useful for structuring multiple sources of variation, including components associated with measured factors, such as covariates and variation attributed to unmeasured factors, or random effects.

The use of random factors is not restricted to linear mixed models. For many reasons, we may want to incorporate random factors into nonlinear models. We may wish to build a model that accommodates correlated data, or to consider the levels of a factor from a selected population in order to make inference about the population. A basic linear model has mean $E(y)=X \beta$. We incorporate random effects by enlarging the model as $E(y \mid u)=X \beta+Z u$. If we 
write a combined model matrix $X^{*}=\left[\begin{array}{ll}\mathrm{X} & \mathrm{Z}\end{array}\right]$ and an enlarged "parameter" vector $\beta^{*}=\left[\begin{array}{ll}\beta^{\prime} & u^{\prime}\end{array}\right]$, we obtain $E(y \mid u)=X^{*} \beta^{*}$.

This suggests a straightforward extension of the generalized linear models. Append the random effects in the form $Z u$ to the linear predictor $X \beta$. This will achieve the two main goals of incorporating correlation and allowing broader inference. In this chapter, we define the generalized linear mixed model (GLMM), explore the consequences of adding random factors and discuss a variety of inferential methods.

\subsection{Structure of the Model}

\subsubsection{Conditional distribution of $y$}

To specify the model we start with the conditional distribution of $y$ given $u$. As in $(2 \cdot 1)$ and $(2 \cdot 2)$, the response vector $y$ is typically, but not necessarily, assumed to consist of conditionally independent elements, each with a distribution with density from the exponential family:

$$
\begin{gathered}
y_{i} \mid u \sim \text { indep. } \quad f_{y_{i} \mid u}\left(y_{i} \mid u\right) \\
f_{y_{i} \mid u}\left(y_{i} \mid u\right)=\exp \left\{\frac{y_{i} \theta_{i}-b\left(\theta_{i}\right)}{a_{i}(\phi)}+c\left(y_{i} ; \phi\right)\right\}
\end{gathered}
$$

From $(2 \cdot 8)$, we know the conditional mean of $y_{i}$ is related to $\theta_{i}$ in $(3 \cdot 1)$. It is a transformation of this mean that we wish to model as a linear model in both fixed and random factors:

$$
\begin{aligned}
& E\left[y_{i} \mid u\right]=b^{\prime}\left(\theta_{i}\right)=\mu_{i} \\
& g\left(\mu_{i}\right)=\theta_{i}=x_{i}^{\prime} \beta+z_{i}^{\prime} u
\end{aligned}
$$


As in chapter $2, g(\cdot)$ is a known function, called the link function, $x_{i}^{\prime}$ is the $\mathrm{i}$-th row of the design matrix $X$ for the fixed effects, and $\beta$ is the fixed effects parameter vector. To that specification we have added $z_{i}^{\prime}$, which is the $i$-th row of the design matrix for the random effects, and $u$, the random effect vector of dimension q. Note that we are using $\mu_{i}$ here to denote the conditional mean of $y_{i}$ given $u$, not the unconditional mean. To complete the specification, we assign a distribution to the random effects:

$$
u \sim f_{U}(u)
$$

In light of the fact that the conditional distribution of $y$ given $u$ is just a notational extension of the generalized linear model, $\mu_{i}$ represents conditional mean.

\subsection{Consequences of Having Random Effects}

\subsubsection{Marginal versus conditional distribution}

We study the aspects of the marginal distributions of $y$ in order to understand what has been assumed for the observed data.

\subsubsection{Mean of $y$}

From (3.2), the marginal mean of $y$ can be obtained as

$$
\begin{aligned}
E\left[y_{i}\right] & =E\left[E\left[y_{i} \mid u\right]\right] \\
& =E\left[\mu_{i}\right] \\
& =E\left[g^{-1}\left(x_{i}^{\prime} \beta+z_{i}^{\prime} u\right)\right] .
\end{aligned}
$$

This cannot, in general, be simplified, due to the nonlinear function $g^{-1}(\cdot)$. To illustrate for a particular $g(\cdot)$, suppose we have a $\log \operatorname{link}$ so that $g(\mu)=\log \mu$ and $g^{-1}(x)=\exp \{x\}$. Then we have 


$$
\begin{aligned}
E\left(y_{i}\right) & =E\left[\exp \left\{x_{i}^{\prime} \beta+z_{i}^{\prime} u\right\}\right] \\
& =E\left\{x_{i}^{\prime} \beta\right\} E\left[\exp \left\{z_{i}^{\prime} u\right\}\right] \\
& =\exp \left\{x_{i}^{\prime} \beta\right\} M_{u}\left(z_{i}\right),
\end{aligned}
$$

where $M_{u}\left(z_{i}\right)$ is the moment generating function of $u$ evaluated at $z_{i}$. Suppose further that $u_{i} \sim N\left(0, \sigma_{u}^{2}\right)$ and that each row of $Z$ has a single entry equal to 1 with all the rest being zero. Then

$$
M_{u}\left(z_{i}\right)=\exp \left\{\sigma_{u}^{2} / 2\right\}
$$

and

$$
E\left[y_{i}\right]=\exp \left\{x_{i}^{\prime} \beta\right\} \exp \left\{\sigma_{u}^{2} / 2\right\}
$$

or

$$
\log E\left[y_{i}\right]=x_{i}^{\prime} \beta+\sigma_{u}^{2} / 2
$$

\subsubsection{Variance of $y$}

To derive the marginal variance of $y$ we use:

$$
\begin{aligned}
\operatorname{var}\left(y_{i}\right) & =\operatorname{var}\left(E\left[y_{i} \mid u\right]\right)+E\left[\operatorname{var}\left(y_{i} \mid u\right)\right] \\
& =\operatorname{var}\left(\mu_{i}\right)+E\left[a_{i}(\phi) v\left(\mu_{i}\right)\right] \\
& =\operatorname{var}\left(g^{-1}\left[x_{i}^{\prime} \beta+z_{i}^{\prime} u_{i}\right]\right)+E\left[a_{i}(\phi) v\left(g^{-1}\left[x_{i}^{\prime} \beta+z_{i}^{\prime} u\right]\right)\right],
\end{aligned}
$$

which again cannot be simplified appreciably without making specific assumptions about the form of $g(\cdot)$.

To illustrate the derivation we assume that we have log link and further assume that the elements of $y$, given $u$, are independent with Poisson distributions. Hence the conditional variance of $y_{i}$ given $u$ is $a_{i}(\phi) \nu\left(\mu_{i}\right)=\mu_{i}$. Using the fact in $(3 \cdot 11)$ gives 


$$
\begin{aligned}
\operatorname{var}\left(y_{i}\right) & =\operatorname{var}\left(\mu_{i}\right)+E\left[\mu_{i}\right] \\
& =\operatorname{var}\left(\exp \left\{x_{i}^{\prime} \beta+z_{i}^{\prime} u\right\}\right)+E\left[\exp \left\{x_{i}^{\prime} \beta+z_{i}^{\prime} u\right\}\right] \\
& =E\left[\left(\exp \left\{2\left(x_{i}^{\prime} \beta+z_{i}^{\prime} u\right)\right\}\right)\right]-\left[E\left(\exp \left\{x_{i}^{\prime} \beta+z_{i}^{\prime} u\right\}\right)\right]^{2}+E\left[\exp \left\{x_{i}^{\prime} \beta+z_{i}^{\prime} u\right\}\right] \\
& =\exp \left\{2 x_{i}^{\prime} \beta\right\}\left(M_{u}\left(2 z_{i}\right)-\left[M_{u}\left(z_{i}\right)\right]^{2}+\exp \left\{-x_{i}^{\prime} \beta\right\} M_{u}\left(\left(z_{i}\right)\right)\right.
\end{aligned}
$$

If we make a further assumption that $u_{i} \sim N\left(0, \sigma_{u}^{2}\right)$ and that each row of $Z$ has a single entry equal to 1 with all the rest being zero, then

$$
\begin{aligned}
\operatorname{var}\left(y_{i}\right) & =\exp \left\{2 x_{i}^{\prime} \beta\right\}\left(\exp \left\{2 \sigma_{u}^{2}\right\}-\exp \left\{\sigma_{u}^{2}\right\}\right)+\exp \left\{x_{i}^{\prime} \beta\right\} \exp \left\{\sigma_{u}^{2} / 2\right\} \\
& \left.=\exp \left\{x^{\prime} \beta_{i}+\sigma_{u}^{2} / 2\right\}\left(\exp \left\{x_{i}^{\prime} \beta\right\} \mid \exp \left\{3 \sigma_{u}^{2} / 2\right\}-\exp \left\{\sigma_{u}^{2} / 2\right\}\right]+1\right) \\
& \left.=E\left[y_{i}\right]\left(\exp \left\{x_{i}^{\prime} \beta\right\} \mid \exp \left\{3 \sigma_{u}^{2} / 2\right\}-\exp \left\{\sigma_{u}^{2} / 2\right\}\right]+1\right)
\end{aligned}
$$

Since the term in parentheses in $(3 \cdot 13)$ is greater than 1 , we see that the variance is larger than the mean.

\subsubsection{Covariance and correlation}

The use of random effects introduces a correlation among observations which have random effects in common. Assuming conditional independence of the elements of $y$, the covariance and correlation between $y_{i}$ and $y_{j}$ are given below:

$$
\begin{aligned}
\operatorname{cov}\left(y_{i}, y_{j}\right) & =\operatorname{cov}\left(E\left[y_{i} \mid u\right] E\left[y_{j} \mid u\right]\right)+E\left[\operatorname{cov}\left(y_{i}, y_{j} \mid u\right)\right] \\
& =\operatorname{cov}\left(\mu_{i}, \mu_{j}\right)+E[0] \\
& =\operatorname{cov}\left(g^{-1}\left[x_{i}^{\prime} \beta+z_{i}^{\prime} u\right] g^{-1}\left[x_{j}^{\prime} \beta+z_{j}^{\prime} u\right]\right) .
\end{aligned}
$$

If we have a log link, this can be evaluated as

$$
\operatorname{cov}\left(y_{i}, y_{j}\right)=\operatorname{cov}\left(\exp \left\{x_{i}^{\prime} \beta+z_{i}^{\prime} u\right\}, \exp \left\{x_{j}^{\prime} \beta+z_{i}^{\prime} \beta\right\}\right)
$$




$$
\begin{aligned}
& =\exp \left\{x_{i}^{\prime} \beta+x_{j}^{\prime} \beta\right\} \operatorname{cov}\left(\exp \left\{z_{i}^{\prime} u\right\}, \exp \left\{z_{j}^{\prime} \beta\right\}\right) \\
& =\exp \left\{x_{i}^{\prime} \beta+x_{j}^{\prime} \beta\right\}\left[M_{u}\left(z_{i}+z_{j}\right)-M_{u}\left(z_{i}\right) M_{u}\left(z_{j}\right)\right]
\end{aligned}
$$

If we further assume that $u \sim N\left(0, \sigma_{u}^{2}\right)$ and that each row of $Z$ has a single entry equal to 1 with all the rest being zero, then

$$
\operatorname{cov}\left(y_{i}, y_{j}\right)=\exp \left\{x_{i}^{\prime} \beta+x_{j}^{\prime} \beta\right\}\left\{\exp \left\{\sigma_{u}^{2}\right\}\left(\exp \left\{z_{i}^{\prime} z_{j} \sigma_{u}^{2}\right\}-1\right)\right]
$$

which is equal to zero if $z_{i}^{\prime} z_{j}=0$ (i.e., if the two observations do not share a random effect) and is positive otherwise (in which case $z_{i}^{\prime} z_{j}=1$ ). From (3.16) and $(3 \cdot 13)$ when $z_{i}^{\prime} z_{j}=1$, we can calculate the correlation after canceling $\exp \left\{x_{i}^{\prime} \beta+x_{j}^{\prime} \beta\right\}$ in the numerator and denominator as:

$$
\begin{aligned}
\operatorname{corr}\left(y_{i}, y_{j}\right) & =\frac{e^{2 \sigma_{u}^{2}}-e^{\sigma_{u}^{2}}}{\sqrt{\left(e^{2 \sigma_{u}^{2}}-e^{\sigma_{u}^{2}}+e^{-x_{i}^{\prime} \beta}+\sigma_{u}^{2} / 2\right)\left(e^{2 \sigma_{u}^{2}}-e^{\sigma_{u}^{2}}+e^{x_{j}^{\prime} \beta}+\sigma_{u}^{2} / 2\right)}} \\
& =\frac{1}{\sqrt{\left(1+\omega e^{-x_{i}^{\prime} \beta}\right)\left(1+\omega e^{-x_{j}^{\prime} \beta}\right)}}
\end{aligned}
$$

where

$$
\omega=1 /\left(e^{3 \sigma_{n}^{2} / 2}-e^{\sigma_{k}^{2} / 2}\right)
$$

\subsection{Likelihood Estimation}

From $(3 \cdot 1),(3 \cdot 2)$ and $(3 \cdot 3)$, the elements of $y$ are conditionally independent, that is

$$
y_{i} \mid u \sim \text { indep. } f_{y_{i / u}}\left(y_{i} \mid u\right)
$$

with

$$
u \sim f_{u}(u \mid \alpha)
$$

and 


$$
E\left(y_{i} \mid u\right)=\mu_{i}
$$

with

$$
g(\mu)=x_{i}^{\prime} \beta+z_{i}^{\prime} u
$$

The likelihood function for GLMMs can be obtained as

$$
\begin{aligned}
& L=\int_{n} f_{y \mid u}(y \mid u) f_{u}(u) d u \\
& =\int\left[\prod_{i=1}^{n} f_{y_{i} \mid u}\left(y_{i} \mid u\right)\right] f_{u}(u) d u,
\end{aligned}
$$

where the integration is over the q-dimensional distribution of $u$. For example, consider the Poisson mixed model with normally distributed random effects:

$$
\begin{gathered}
y_{i j} \mid u \sim \text { indep. Poisson }\left(\mu_{i j}\right), \quad(i=1,2, \ldots . . m ; j=1,2, \ldots n i) ; \\
\log \left(\mu_{i j}\right)=x_{i j}^{\prime} \beta+u_{i} \\
u_{i} \sim \text { i.i.d. } N\left(0, \sigma_{u}^{2}\right) .
\end{gathered}
$$

This uses a log-link function and a normal distribution for the random cluster effects. For model $(3 \cdot 19)$, the log-likelihood is

$$
\begin{aligned}
& l=\log \left[\prod_{i=1}^{m} \int_{-\infty}^{+\infty} f_{y_{i} \mid u}\left(y_{i} \mid u_{i}\right) f_{u}\left(u_{i}\right) d u_{i}\right] \\
& =\log \left[\prod_{i=1}^{m} \int_{-\infty}^{+\infty}\left[\prod_{j=1}^{n i} f_{y_{i j} \mid u_{i}}\left(y_{i j} \mid u_{i}\right)\right] f_{u}\left(u_{i}\right) d u_{i}\right] \\
& =\log \left[\prod_{i=1}^{m} \int_{-\infty}^{+\infty}\left[\prod_{j=1}^{n i} \frac{e^{\mu_{i j}} \mu_{i j}^{y_{i j}}}{y_{i j} !}\right] f_{u}\left(u_{i}\right) d u_{i}\right] \\
& =\sum_{i=1}^{m} \log \left[\int_{-\infty}^{+\infty} \frac{e^{-\left(-\sum_{j=1}^{n_{i}} \mu_{i j}+\sum_{j=1}^{n_{i}} y_{i} \log \mu_{i j}\right)}}{\prod\left(y_{i j} !\right)} f_{u}\left(u_{i}\right) d u_{i}\right]
\end{aligned}
$$




$$
\begin{aligned}
& =\sum_{i=1}^{m} \log \left[\frac{1}{\prod_{j=1}^{n_{i}}\left(y_{i j}\right)} \int_{-\infty}^{+\infty} e^{-\sum_{j=1}^{n_{i}} \mu_{i j}+\sum_{j=1}^{n_{i}} y_{i j}\left(x_{i j} \beta+u_{i}\right)} f_{u}\left(u_{i}\right) d u_{i}\right] \\
& =\sum_{i=1}^{m}\left[-\sum_{j=1}^{n_{i}} \log \left(y_{i j} !\right)+\sum_{j=1}^{n} y_{i j} x_{i j}^{\prime} \beta\right]+\sum_{i=1}^{m} \int_{-\infty}^{+\infty}\left[e^{-\sum_{j} \mu_{i j}^{\prime}+\sum_{j} y_{i j} u_{i}}\right] f_{u}\left(u_{i}\right) d u_{i} \\
& =y^{\prime} X \beta-\sum_{i j} \log \left(y_{i j} !\right)+\sum_{i=1}^{m} \int_{-\infty}^{+\infty}\left[e^{-\sum_{j} \mu_{y}+u_{i} y_{i}}\right] f_{u}\left(u_{i}\right) d u_{i}
\end{aligned}
$$

Unfortunately, $(3 \cdot 20)$ cannot be simplified further or evaluated in closed form and hence maximizing values cannot be expressed in closed form either.

In the simplest case, numerical integration for calculating the likelihood is straightforward and hence numerical maximization of the likelihood is not too difficult. For example, for $(3 \cdot 19)$ as seen in $(3 \cdot 20)$, the log likelihood is the sum of independence contributions from each cluster, each of which involves just a single- dimensional integral. This integral can be evaluated accurately using standard quadrature techniques, Gauss-Hermite quadrature discussed in chapter-4. This "brute force" approach to maximum likelihood works relatively well in simple situations: a single random effect, and random effects which come in clusters (e.g., longitudinal data with subjects having random intercepts and slopes). However, for more complicated structures (e.g., crossed random factors) it fails.

\subsubsection{Fixed effects parameters}

Even through the likelihood equations are numerically difficult, we can write them in simpler form. From $(3 \cdot 18)$, we get,

$$
l=\log \int f_{y \mid u}(y \mid u) f_{u}(u) d u=\log f_{y}(y)
$$

so that 


$$
\begin{aligned}
\frac{d l}{d \beta} & =\frac{\partial}{\partial \beta} \int f_{y \mid u}(y \mid u) f_{u}(u) d u / f_{y}(y) \\
& =\int\left[\frac{\partial}{\partial \beta} f_{y \mid u}(y \mid u)\right] f_{u}(u) d u / f_{y}(y)
\end{aligned}
$$

since $f_{u}(u)$ does not involve $\beta$. Noting that

$$
\begin{aligned}
\frac{\partial}{\partial \beta} \int f_{y \mid u}(y \mid u) & =\left(\frac{1}{f_{y \mid u}(y \mid u)} \cdot \frac{\partial f_{y \mid u}(y \mid u)}{\partial \beta}\right) f_{y \mid u}(y \mid u) \\
& =\frac{\partial \log f_{y \mid u}(y \mid u)}{\partial \beta} f_{y \mid u}(y \mid u)
\end{aligned}
$$

we can write $(3 \cdot 22)$ as

$$
\begin{aligned}
\frac{\partial l}{\partial \beta} & =\int \frac{\partial \log f_{y \mid u}(y \mid u)}{\partial \beta} f_{y \mid u}(y \mid u) f_{U}(u) d u / f_{y}(y) \\
& =\int \frac{\partial \log f_{y \mid u}(y \mid u)}{\partial \beta} f_{u \mid y}(u \mid y) d u .
\end{aligned}
$$

Using $(2 \cdot 20)$ which gives the derivative of the log likelihood for a GLM $(3-24)$ gives

$$
\begin{aligned}
& \frac{d l}{d \beta}=\int X^{\prime} W^{*}(y-\mu) f_{u \mid y}(u \mid y) d u \\
& =X^{\prime} E\left[W^{*} \mid y\right]-X^{\prime} E\left[W^{*} \mu \mid y\right]
\end{aligned}
$$

where

$$
W^{*}=\left\{\left[a_{i}(\phi) v\left(\mu_{i}\right) g_{\mu}\left(\mu_{i}\right)\right]^{-1}\right\}
$$

The likelihood equation for $\beta$ is therefore,

$$
X^{\prime} E\left[W^{*} \mid y\right]=X^{\prime} E\left[W^{*} \mu \mid y\right]
$$




\subsubsection{Random effects parameters}

A result similar to $(3 \cdot 24)$ can be derived for the $M L$ equations for the parameters in the distribution of $f_{u}(u)$. Let $\varphi$ denote those parameters, so that

$$
\begin{aligned}
\frac{\partial l}{\partial \varphi} & =\int \frac{\partial \log f_{u}(u)}{\partial \varphi} f_{u \mid y}(u \mid y) d u \\
& =E\left[\frac{\partial \log f_{u}(u)}{\partial \varphi} \mid y\right] .
\end{aligned}
$$

Further simplifications are not possible without specifying a form for the random effects distribution.

\subsection{Penalized Quasi-likelihood}

For the generalized linear models, the working variates and the principal of quasi- likelihood are highly useful concepts. Working variates form the basis of efficient computing algorithms for both maximum likelihood and maximum quasi-likelihood. These working variates can be used in generalized linear mixed models (GLMMs). Working variates for generalized linear models begin with a Taylor series expansion of the link function around the mean $y_{i}$ :

$$
Z_{i}=x_{i}^{\prime} \beta+g_{\mu}\left(\mu_{i}\right)\left(y_{i}-\mu_{i}\right)
$$

The working variates thus follows a linear model and can be used to form a provisional estimate of $\beta$. The direct analog of working variates for the GLMM specification in (3.1) and (3.2) would be an expansion around the conditional mean of $y_{i}$ :

$$
Z_{i}=x_{i}^{\prime} \beta+Z_{i}^{\prime} u+g_{\mu}\left(\mu_{i}\right)\left(y_{i}-\mu_{i}\right)
$$

or, in matrix notation

$$
Z=X \beta+Z u+\Delta(y-\mu)
$$


where

$$
\Delta=\left\{g_{\mu}\left(\mu_{i}\right)\right\}
$$

To derive a local approximation, the next step would be to calculate the variance of $Z$. But this approximation quickly becomes complicated, since $\Delta$ and $\mu=E(y \mid u)$ itself are random functions of $\mathrm{u}$ and their variances cannot be easily obtained. A possible simplification is made by simplifying $(3 \cdot 30)$ to be

$$
Z=X \beta+Z u+\Delta^{*}(y-\mu)
$$

where

$$
\Delta^{*}=\left\{{ }_{d} g_{\mu}\left[g^{-1}\left(x_{i}^{\prime} \beta\right)\right]\right\}
$$

Under the simplification, $\operatorname{var}(Z)=Z D Z^{\prime}+\Delta^{*} \operatorname{var}(y-\mu) \Delta^{*}$

$$
\equiv Z D Z^{\prime}+R
$$

where

$$
\mathrm{R}=\Delta^{*} \operatorname{var}(y-\mu) \Delta^{*}
$$

That is, the working variate $Z$ approximately follows a linear mixed model (LMM). This suggests an iterative algorithm (Schall, 1991) in which an LMM is fitted to get estimates of $\beta$ and $u$.

\subsubsection{Likelihood Estimation}

A completely different justification of the above approach is via what is called penalized quasi-likelihood (PQL). The quasi-likelihood does not specify a distribution, only the mean-to-variance relationship. This is not a sufficient basis on which to estimate the variance-covariance structure. To remedy this 
defect, Green and Silverman (1994) suggested to add a penalty function to the quasi-likelihood of the form $\frac{1}{2} u^{\prime} D^{-1} u$, that is $P Q L=\sum Q_{i}-\frac{1}{2} u^{\prime} D^{-1} u$ where

$$
Q_{i}=\int_{y_{i}}^{\mu_{i}} \frac{y_{i}-t}{\sigma^{2} v(t)} d t
$$

The attractive features of quasi-likelihood, namely model robustness and less restrictive assumptions, have led to a search for generalizations applicable to GLMMs. Central of these is to used Laplace approximation (Tierney and Kadane, 1986) for evaluating the high- dimensional integral in the likelihood. The basic form of Laplace's approximation is based on second-order Taylor series expansion and takes the form

$$
\log \int e^{h(u)} d u=h\left(u_{0}\right)+\frac{q}{2} \log 2 \pi-\frac{1}{2} \log \left|-\frac{\partial^{2} h(u)}{\partial u \partial u^{\prime}}\right|
$$

where, $u_{0}$ is the solution to

$$
\left.\frac{\partial h(u)}{\partial u}\right|_{u=u_{0}}=0
$$

We utilize the result to approximate the log likelihood of the GLMM via

$$
\begin{aligned}
& l=\log \int f_{y \mid U} f_{U} d u \\
& =\log \int e^{\log f_{y \mid U^{+}}+\log f_{U}} d u \\
& =\log \int e^{\log _{y \mid U}+\log f_{U}} d u
\end{aligned}
$$

with

$$
h(u)=\log f_{Y \mid U}+\log f_{U}
$$


To construct the Laplace approximation, (3.35) must be solved and an expression for $\frac{\partial^{2} h(u)}{\partial u \partial u^{\prime}}$ is needed. If we assume that $u \sim N(0, D)$ then

$$
\log f_{U}=-\frac{1}{2} u^{\prime} D^{-1} u-\frac{q}{2} \log 2 \pi-\frac{1}{2} \log |D|
$$

and $h(u)$ becomes

$$
\log f_{Y \mid U}+\log f_{U}=\log f_{Y \mid U}-\frac{1}{2} u^{\prime} D^{-1} u-\frac{q}{2} \log 2 \pi-\frac{1}{2} \log |D|
$$

Differentiating with respect to $u$ gives

$$
\begin{aligned}
\frac{\partial h(u)}{\partial u} & =\frac{\partial \log f_{Y \mid U}}{\partial u}-D^{-1} u \\
& =\frac{1}{\sigma^{2}} Z^{\prime} W \Delta(y-\mu)-D^{-1} u
\end{aligned}
$$

The second equality comes from derivatives identical to $(2 \cdot 19)$ with $u$ replacing $\beta$ and $Z$ replacing $X$. To find $u_{0}$ it is necessary to solve for $u$ from

$$
\frac{1}{\sigma^{2}} Z^{\prime} W \Delta(y-\mu)=D^{-1} u
$$

which is not as simple as it appears since $W, \Delta$, and $\mu=E[y \mid u]$ on the lefthand side of the equation are all functions of $u$. We will need the second derivative:

$$
\frac{\partial^{2} h(u)}{\partial u \partial u^{\prime}}=-\frac{1}{\sigma^{2}} Z^{\prime} W \Delta \frac{\partial \mu}{\partial u^{\prime}}+\frac{1}{\sigma^{2}} Z^{\prime} \frac{\partial W \Delta}{\partial u^{\prime}}(y-\mu)-D^{-1}
$$

For binomial and Poisson regression, $W \Delta=I, \mathrm{I}$ is identity matrix, so the second term on the right side is zero. In general, the second term has expectation zero 
with respect to the conditional distribution of $y$ given $u$. So it may be reasonable to consider it as negligible with respect to the other terms. If this is the case $(3.40)$ becomes

$$
\begin{aligned}
-\frac{\partial^{2} h(u)}{\partial u \partial u^{\prime}} & =\frac{1}{\sigma^{2}} Z^{\prime} W \Delta \Delta^{-1} Z+0+D^{-1} \\
& =\frac{1}{\sigma^{2}} Z^{\prime} W Z+D^{-1} \\
& =\left(\frac{1}{\sigma^{2}} Z^{\prime} W Z D+I\right) D^{-1}
\end{aligned}
$$

Using (3.41) in (3.34) gives

$$
\begin{gathered}
l=\log f_{Y \mid U}\left(y \mid u_{0}\right)-\frac{1}{2} u_{0}^{\prime} D^{-1} u-\frac{q}{2} \log 2 \pi-\frac{1}{2} \log |D|+\frac{q}{2} \log 2 \pi-\frac{1}{2} \log \left(Z^{\prime} W Z D / \sigma^{2}+1\right) D^{-1} \mid \\
=\log f_{Y \mid U}\left(y \mid u_{0}\right)-\frac{1}{2} u_{0}^{\prime} D^{-1} u+\frac{1}{2} \log \left(Z^{\prime} W Z D / \sigma^{2}+1\right)
\end{gathered}
$$

This still must be maximized with respect to $\beta$ to find the maximum likelihood estimate. Differentiating with respect to $\beta$ gives an approximate score equation of

$$
\begin{aligned}
\frac{\partial l}{\partial \beta}= & \frac{\partial \log f_{Y / U}\left(y \mid u_{0}\right)}{\partial \beta}+\frac{\partial}{\partial \beta} \frac{1}{2}\left|Z^{\prime} W Z D / \sigma^{2}+I\right| \\
= & \frac{1}{\sigma^{2}} X^{\prime} W \Delta(y-\mu)+\frac{\partial}{\partial \beta} \frac{1}{2}\left|Z^{\prime} W Z D / \sigma^{2}+I\right| \\
= & \frac{1}{\sigma^{2}} X^{\prime} W \Delta(y-\mu),
\end{aligned}
$$

where the second equality follows $(2 \cdot 19)$ and for the third we have assumed that $W$ changes negligibly as a function of $\beta$. Thus, the maximum quasilikelihood equations for $\beta$ and $u$ are obtained as 


$$
\frac{1}{\sigma^{2}} X^{\prime} W \Delta^{*}(y-\mu)=0
$$

and

$$
\frac{1}{\sigma^{2}} Z W \Delta^{*}(y-\mu)-D^{-1} u=0
$$

Equations (3.44) and (3.45) can also arise from jointly maximizing $\log f_{Y \mid U}-\frac{1}{2} u^{\prime} D^{-1} u$ with respect to $\beta$ and $u$ which is similar to a quasilikelihood with a "penalty" function added on the term $u^{\prime} D^{-1} u$. In (3.45) the $\frac{1}{2} u^{\prime} D^{-1} u$ term serves to prevent arbitrary values of $u$ from being selected and forces to be closer to zero. Methods to solve these equations are thus frequently called penalized quasi likelihood (PQL) methods. These lead (Breslow and Clayton, 1993) to a computation algorithm similar to that of Schall (1991), Green (1990), and Wolffinger (1993).

\subsubsection{Variance Component Estimation}

Breslow and Clayton (1993) made some further approximations to motivate standard estimating equations in terms of working vector $Y$, the iterated weights $W$, and the design matrices $X$ and $Z$. Ignoring throughout the dependence of $\mathrm{W}$ on $\theta$ and replacing the deviance $\sum d_{i}\left(y_{i}, \mu_{i}^{b}\right)$ by the Pearson chi-squared statistic $\sum\left(y_{i}-\mu_{i}^{b}\right)^{2} /\left[a_{i} v\left(\mu_{i}^{b}\right)\right]$, and by the usual additive constant

$$
q l(\hat{\alpha}(\theta), \theta) \approx-\frac{1}{2} \log |V|-\frac{1}{2}(Y-X \hat{\alpha})^{\prime} V^{-1}(Y-X \hat{\alpha})
$$

We use in practice the REML version (Patterson and Thomson 1971):

$$
q l_{1}(\hat{\alpha}(\theta), \theta) \approx-\frac{1}{2} \log |V|-\frac{1}{2} \log \left|X^{\prime} V^{-1} X\right|-\frac{1}{2}(Y-X \hat{\alpha})^{\prime} V^{-1}(Y-X \hat{\alpha}) . \cdots(3
$$


For the normal theory linear model this adjustment corresponds to the profile likelihood correction of Cox and Reid (1987). Following Harville (1977), we define $P=V^{-1}-V^{-1} X \times\left(X^{\prime} V^{-1} X\right) X^{\prime} V^{-1}$ and differentiate (3.47) with respect to components $\theta$ to obtain estimating equations for the variance parameters:

$$
-\frac{1}{2}\left[(Y-X \alpha)^{\prime} V^{-1} \frac{\partial V}{\partial \theta_{j}} V^{-1}(Y-X \alpha)-\operatorname{tr}\left(P \frac{\partial V}{\partial \theta_{j}}\right)\right]=0
$$

The corresponding Fisher information matrix has the components

$$
I_{j k}=-\frac{1}{2} \operatorname{tr}\left(P \frac{\partial V}{\partial \theta_{j}} P \frac{\partial V}{\partial \theta_{k}}\right)
$$

\subsection{Computing ML Estimates}

\subsubsection{Numerical quadrature}

Generalized linear mixed models pose special challenges beyond linear mixed models because of the high dimensional integration required to evaluate (and hence maximize) the likelihood. We start by considering a GLMM with a single, normally distributed random effect. Let $y_{i j}$ be the $\mathrm{j}$-th observation corresponding to the $\mathrm{i}$-th level of the random effect so that

$$
\begin{gathered}
y_{i j} \mid u \sim \text { indep. } f_{Y_{i j \mid u}}\left(y_{i j} \mid u\right) \\
f_{Y_{i j \mid u}}\left(y_{i j} \mid u\right)=\exp \left\{\left[y_{i j} \theta_{i j}-b\left(\theta_{i j}\right)\right] / a_{i}(\phi)+c\left(y_{i j}, \phi\right)\right\} \\
E\left[y_{i j} \mid u\right]=\mu_{i j} \\
g\left(\mu_{i j}\right)=x_{i j}^{\prime} \beta+u_{i}
\end{gathered}
$$

with 


$$
u_{i} \sim \text { i.i.d. } . N\left(0, \sigma_{u}^{2}\right)
$$

The likelihood for the model is

$$
\begin{aligned}
& L=\int \prod_{i j} f_{y_{i j i k}}\left(y_{i j} \mid u_{i}\right) f_{u_{i}}\left(u_{i}\right) d u_{i} \\
& =\prod_{i} \int_{-\infty}^{+\infty} e^{\sum_{j}\left[\left(v_{i j} \theta_{i j}-b\left(\theta_{i j}\right)\right] / a_{i}(\phi)-\sum_{j} c\left(y_{i, \phi}, \phi\right)\right.} \frac{e^{-u_{i}^{2} /\left(2 \sigma_{u}^{2}\right)}}{\sqrt{2 \pi \sigma_{u}^{2}}} d u_{u}, \\
& =\prod_{i} \int_{-\infty}^{+\infty} h_{i}\left(u_{i}\right) \frac{e^{-u_{i}^{2} /\left(2 \sigma_{u}^{2}\right)}}{\sqrt{2 \pi \sigma_{u}^{2}}} d u_{i},
\end{aligned}
$$

where

$$
h_{i}\left(u_{i}\right)=e^{\sum_{j}\left|y_{i j} \theta_{i j}-b\left(s_{i j}\right)\right| a_{i}(\phi)-\Sigma_{j} c\left(y_{i j}, \phi\right)} \text { and } \theta_{i j} \text { is a function of } u_{i}
$$

It can be seen that the likelihood is the product of one-dimensional integrals of the form

$$
\int_{-\infty}^{+\infty} h(u) \frac{e^{-u^{2} /\left(2 \sigma_{u}^{2}\right)}}{\sqrt{2 \pi \sigma_{u}^{2}}} d u
$$

which, upon a change of variables of $u=\sqrt{2} \sigma_{u} v$, can be written as

$$
\int_{-\infty}^{+\infty} h\left(\sqrt{2} \sigma_{u} v\right) \frac{e^{-v^{2}}}{\sqrt{\pi}} d v \equiv \int_{-\infty}^{+\infty} h^{*}(v) e^{-v^{2}} d v
$$

where

$$
h^{*}(.) \equiv h\left(\sqrt{2} \sigma_{u}\right) / \sqrt{\pi}
$$

\subsubsection{Gauss-Hermite quadrature}

Numerical integration over an unbounded range can be difficult. However, for integrals of smooth functions $h^{*}($.$) multiplied by the function e^{-v^{2}}$, the method 
of Gauss-Hermite-quadrature is available. This approximates the integral in $(3 \cdot 52)$ as a weighted sum:

$$
\int_{-\infty}^{+\infty} h^{*}(v) e^{-v^{2}} d v \cong \sum_{k=1}^{d} h^{*}\left(x_{k}\right) w_{k},
$$

where the weights $w_{k}$ and the evaluation points $x_{k}$ are designed to provide an accurate approximation in the case where $h^{*}(\cdot)$ is a polynomial. Abramowitz and Stegun (1964), calculated $x_{k}$ and $w_{k}$ in the following forms

$$
\begin{aligned}
x_{k} & =\mathrm{i} \text {-th zero of } H_{n}(x) \\
w_{k} & =\frac{2^{n-1} n ! \sqrt{\pi}}{n^{2}\left[H_{n-1}\left(x_{k}\right)\right]^{2}}
\end{aligned}
$$

where $H_{n}(x)$ is the Hermite polynomial of degree $n$. By using quadrature of a high-enough degree, accurate approximation can be calculated to integrals of functions that are similar to those of any high-degree polynomial.

\subsubsection{Likelihood calculations}

Gauss-Hermite quadrature can be used to calculate integrals with respect to the normal density as

$$
\int_{-\infty}^{+\infty} h(x) \frac{e^{-x^{2} /\left(2 \sigma^{2}\right)}}{\sqrt{2 \pi \sigma^{2}}} d x \cong \sum_{k=1}^{d} h\left(\sqrt{2} \sigma x_{k}\right) w_{k} / \sqrt{\pi} .
$$

To derive an approximation to a likelihood such as (3.51) and (3.55), would be used repeatedly. For example, suppose that our model was, for $i=1,2, \ldots, m$ and $j=1,2, \ldots ., n$,

$$
\begin{gathered}
y_{i j} \mid u \sim \text { indep. Bernoulli }\left(p_{i j}\right) \\
\operatorname{logit}\left(p_{i j}\right)=\mu+a_{i} \text { and, } a_{i} \sim \text { i.i.d.N }\left(0, \sigma_{a}^{2}\right)
\end{gathered}
$$


The log likelihood would be

$$
\begin{aligned}
& l=\sum_{i} \log \int_{-\infty}^{+\infty} e^{\left(\mu+a_{i}\right) y_{i}-n \log \left(1+e^{\mu+a_{i}}\right)} \frac{e^{-a_{i}^{2} /\left(2 \sigma_{a}^{2}\right)}}{\sqrt{2 \pi \sigma_{a}^{2}}} d a_{i} \\
& \cong \sum_{i} \log \left(\sum_{k} e^{\left(\mu+x_{k}\right) y_{i} \cdot-n \log \left(1+e^{\mu+x_{k}}\right)} w_{k} / \sqrt{\pi}\right) .
\end{aligned}
$$

This log likelihood needs to be maximized numerically to get estimates of $\mu$ and $\sigma_{a}$. Derivatives of the $\log$ likelihood, which are often required by numerical maximization algorithms, can be approximated similarly. Alternatively, quasiNewton or derivative-free maximization methods can be used.

A likelihood ratio test, or best predicted values, would require similar numerical calculation. For example, the best predicted values for model $(3 \cdot 56)$ are

$$
\begin{aligned}
& E\left[a_{i} \mid y\right]=\int a_{i} f_{a_{i} \mid y}\left(a_{i} \mid y\right) d a_{i} \\
& \frac{\int a_{i} e^{\left(\mu+x_{k}\right) y_{i}-n \log \left(1+e^{\mu+x_{k}}\right)} f_{a_{i}}\left(a_{i}\right) d a_{i}}{\int e^{\left(\mu+x_{k}\right) y_{i}-n \log \left(1+e^{\mu+x_{k}}\right)} f_{a_{i}}\left(a_{i}\right) d a_{i}} .
\end{aligned}
$$

The denominator is exactly the likelihood, the approximation for which is displayed in (3.57), and the numerator would be approximated similarly. If the MLEs $\hat{\mu}$ and $\hat{\sigma}_{a}$ were used in the calculation, then the approximation would be for the estimated best predictor. 


\subsubsection{Monte Carlo EM (MCEM)}

We return to GLMM as our most general model

$$
\begin{gathered}
y_{i} \mid u \sim \operatorname{indep} . f_{Y_{i} \mid U}\left(y_{i} \mid u\right) \\
f_{Y_{i \mid u}}\left(y_{i} \mid u\right)=\exp \left\{\left[y_{i} \theta_{i}-b\left(\theta_{i}\right)\right] / a_{i}(\phi)+c\left(y_{i}, \phi\right)\right\} \\
E\left[y_{i} \mid u\right]=\mu_{i} \\
g\left(\mu_{i}\right)=x_{i}^{\prime} \beta+u_{i} \\
u \sim f_{U}(u \mid D),
\end{gathered}
$$

where $D$ is the parameters governing the distribution of $u$. To set up the EM algorithm, consider the random effects, $u$, to be missing data. The complete data, $\mathrm{W}$, is then $\mathrm{W}=(\mathrm{Y}, \mathrm{u})$, and the complete data log-likelihood is given by

$$
\ln L_{W}=\sum_{i} \ln f_{y_{i} \mid u}\left(y_{i} \mid u, \beta, \phi\right)+\ln f_{u}(u \mid D)
$$

This choice of missing data has two advantages. First, on knowing the u's, the $Y_{i}$ 's are independent. Second, the $M$ step of the EM algorithm maximizes (3.60) with respect to $\beta, \phi$ and D. Because $\beta$ and $\phi$ enter only in the first term, the $\mathrm{M}$ step with respect to $\beta$ and $\phi$ uses only $f_{y \mid u}$, and so it is similar to a standard generalized linear model computation with the values of $u$ treated as known.

The EM algorithm then takes the following form:

1. Choose starting values $\beta^{(0)}, \phi^{(0)}$, and $D^{(0)}$. Set $\mathrm{m}=0$.

2. Calculate (with expectations evaluated under $\beta^{(m)}, \phi^{(m)}$ and $D^{(m)}$ :
a. $\beta^{(m+1)}$ and $\phi^{(m+1)}$, which maximizes $E\left[\ln f_{y \mid u}(y \mid u, \beta, \phi) \mid u\right]$
b. $D^{(m+1)}$ which maximizes $E\left[\ln f_{u}(u \mid D) \mid y\right]$
c. Set $\mathrm{m}=\mathrm{m}+1$.

3. If converges is achieved, declare $\beta^{(m+1)}, \phi^{(m+1)}$ and $D^{(m+1)}$ to be maximum likelihood estimators (MLE's); otherwise, return to step 2. 


\subsubsection{Monte Carlo Newton -Raphson (MCNR)}

There is also a simulation analog of working variates or Fisher scoring approach which was used to fit GLMs. Whenever the marginal density of $y$ is formed as a mixture in (3.59) with separate parameters for $f_{Y \mid U}$ and $f_{U}$, then the $\mathrm{ML}$ equations for $\theta=\left(\beta^{\prime}, \phi\right)^{\prime}$ and $D$ take the following form:

$$
E\left[\frac{\partial f_{Y \mid U}(y \mid u, \theta)}{\partial \theta} \mid y\right]=0
$$

and

$$
E\left[\frac{\partial f_{U}(u \mid D)}{\partial D} \mid y\right]=0
$$

Equation (3.62) involves only the distribution of $u$ and is often fairly easy to solve, e.g., when the distribution is normal. On the other hand, (3.61) is amenable to Newton-Raphson or a scoring approach. Expanding $\partial f_{Y \mid U}(y \mid u, \theta) / \partial \beta$ as a function of $\beta$ around a value $\theta_{0}$ gives

$$
\left.\frac{\partial f_{Y \mid U}(y \mid u, \theta)}{\partial \beta} \cong \frac{\partial f_{Y \mid U}(y \mid u, \theta)}{\partial \beta}\right|_{\theta=\theta_{0}}+\left.\frac{\partial^{2} f_{Y \mid U}(y \mid u, \theta)}{\partial \beta \partial \beta^{\prime}}\right|_{\theta=\theta_{0}}\left(\beta-\beta_{0}\right)
$$

Specializing this to our model, and dropping the term with a conditional expected value of zero, the formula for a scoring-type algorithm becomes

$$
\frac{\partial f_{Y / U}(y \mid u, \theta)}{\partial \beta} \cong \frac{1}{\phi} X^{\prime} W \Delta(y-\mu)-\frac{1}{\phi} X^{\prime} W X\left(\beta-\beta_{0}\right)
$$

where

$$
W=\left\{\left[v\left(\mu_{i}\right) g_{\mu}^{2}\left(\mu_{i}\right)\right]^{-1}\right\} \text { and } \Delta=\left\{g_{\mu}\left(\mu_{i}\right)\right\}
$$


and it is clear that $W, \Delta$ and $\mu=E[y \mid u]$ are all functions of $u$ and that all parameters are evaluated as $\theta=\theta_{0}$. Using this approximation in (3.61) leads to an iteration equation of the form

$$
\beta^{(m+1)}=\beta^{(m)}+\left(X^{\prime} E[W \mid y] X\right)^{-1} X^{\prime} E[W \Delta(y-\mu) \mid y] .
$$

This analog of scoring would proceed by iteratively solving (3.64), (3.62) and an equation for $\phi$.

\subsubsection{Simulated maximum likelihood}

Although both MCEM and MCNR work on the log of likelihood, Geyer and Thompson (1992), Gelfand and Carlin (1993), Durbin and Koopman (1997) have suggested simulation to estimate the value of the likelihood directly. Starting from the likelihood, we have

$$
\begin{aligned}
L & =\int_{Y \mid U}(y \mid u, \beta, \phi) f_{U}(u \mid D) d u \\
& =\int \frac{f_{Y \mid u}(y \mid u, \beta, \phi) f_{U}(u \mid D)}{h_{U}(u)} h_{U}(u) d u \\
& =E_{U}\left[\frac{f_{Y \mid U}(y \mid u, \beta, \phi) f_{U}(u \mid D)}{h_{U}(u)}\right] \\
& \cong \frac{1}{N} \sum_{k=1}^{N} \frac{f_{Y \mid U}\left(y \mid u^{(k)}, \beta, \phi\right) f_{U}\left(u^{(k)} \mid D\right)}{h_{U}\left(u^{(k)}\right)},
\end{aligned}
$$

where the subscript $u$ on the expectation is a reminder that the expectation is with respect to $u, h_{U}(u)$ is a density with respect to which the expectation is taken, $u^{(k)}$ are selected from this density, and $N$ is the number of simulated values. This is an unbiased estimate no matter the choice of $h_{U}(u)$. The simulated likelihood is then is then numerically maximized, either after a 
single simulation, or using multiple simulations in an iterative process, where the importance of sampling distribution is allowed to depend on the current parameter values. Although unbiased, the approximation is sensitive to the choice of $h_{U}(u)$ in the sense that it can be highly variable for choices far from the optimal choice. 


\section{Chapter 4}

\section{Misspecified Generalized Linear}

\section{Mixed Model}

\subsection{Introduction}

Maximum likelihood (ML) is a commonly used method for analyzing generalized linear mixed models. The ML estimates are the most efficient under the assumption that the models are correctly specified. However, for an incorrectly specified regression model, the ML method generally provides biased estimates.

The impact of model violations on the estimate of a regression coefficient in a generalized linear mixed model was investigated by a number of authors. Neuhaus et al. (1992) considered logistic-normal models and a misspecified mixing distribution. They concluded that, although regression estimates are asymptotically biased, the magnitude of bias is typically small. Liang and Hanfelt (1994) considered likelihood inference using the beta binomial model and showed that ignoring variation in the within-cluster correlation as a 
function of covariates can result in severe bias for regression estimates. Molenberghs et al. (1998) studied the performance of test statistics based on misspecified models. Ten Have et al. (1999) studied binary data with multiple levels of clustering and showed that fitting an incomplete multilevel structure can lead to bias. Tan et al. (1999) showed that using generalized estimating equations (Liang \& Zeger, 1986) for an underlying latent variable model does not lead to biased estimation or inference. Palta et al. (1997) presented a general study of omitted confounders and other forms of misspecification when models are fitted using generalized estimating equations.

For the linear mixed model the selection of a proper random effects structure is necessary for efficient estimation and for proper model-based standard error estimates. Misspecification of the covariance does not affect the consistency of the maximum likelihood regression estimator. For generalized linear mixed models, the use of non-linear link functions means the proper selection of the random effects structure may be required both for valid point estimates and for correct standard error. Tan Have et al. (1999), Heagerty (1999), Heagerty \& Zeger (2000) and Heagerty and Kurland (2001) illustrate that generalized linear mixed model estimates can be sensitive to the random effect assumptions.

In this thesis, we study the impact of misspecification of the random effects assumptions on the commonly-used MLand PQL methods for fitting generalized linear mixed models. We ran a number of simulations by considering various types of random effects assumptions. In all cases, we fit the data assuming a misspecified Gaussian random intercept term for the GLMM's. We explore-the simulated biases and root mean squared errors of the ML and PQL estimates arising from incorrectly specified regression models. 


\subsection{Asymptotic Bias of ML estimates for Misspecified Models}

General theoretical results for misspecified maximum likelihood estimators were presented by White (1982). Heagerty and Kurkland (2001) investigated the asymptotic behavior of the maximum likelihood estimators for both conditionally specified and marginally specified generalized linear mixed models.

These authors studied a special case where the random effect $u_{i}$ is assumed to follow an incorrectly specified distribution $f_{u_{i}}\left(u_{i} \mid \alpha\right)$. If the true distribution of $u_{i}$ is $g_{u_{i}}\left(u_{u} \mid \theta_{0}\right)$, then the misspecified maximum likelihood estimators $\hat{\theta}_{N}=\left(\hat{\beta}_{n}, \hat{\alpha}_{n}\right)$ converge to the value $\theta^{*}$ that minimizes the Kullback-Leibler information criteria. Equivalently, $\theta^{*}$ is obtained by solving the equation

$$
\lim E_{\theta_{0}}\left\{\left.\sum_{i=1}^{N} \frac{\partial}{\partial \theta} \log f_{\theta}\left(Y_{i} / X_{i}\right)\right|_{\theta^{*}}\right\}=0,
$$

where the expectation is taken with respect to the true model. White (1992) showed that $\sqrt{N}\left(\hat{\theta}_{N}-\theta^{*}\right)$ is asymptotically normally distributed with mean 0 and a certain covariance matrix. Heagerty and Kurland (2001) evaluate the asymptotic relative bias $100^{*}\left(\theta^{*}-\theta_{0}\right) / \theta_{0}$ that results from incorrect assumption regarding the random effects in-the generalized linear mixed models.

In the next section, we discuss various types of random effects distributions commonly used in generalized linear mixed models. 


\subsection{Illustrative example:}

\section{A binary logistic model}

Given the random effects $u_{i j}$, assume that the response $y_{i j}$ is binary with mean $\mu_{i j}$ where

$$
\mathrm{g}\left(\mu_{i j}\right)=\operatorname{logit}\left(\mu_{i j}\right)=\beta_{0}+\beta_{1} X_{i j, 1}+\beta_{2} X_{i j, 2}+\beta_{3} X_{i j, 1} X_{i j, 2}+u_{i j}
$$

where

$$
X_{i j, 1} \text { is the cluster-level covariate }
$$

and

$$
X_{i j, 2} \text { is the within-cluster covariate }
$$

with $X_{i j, 1}=x_{i}$ taking the value 0 or 1 and with $X_{i j, 2}=x_{j}=(j-1) /\left(n_{i}-1\right)$ for $\mathrm{j}=1$, $2,3, \ldots, n_{i}$.

We also consider the interaction between the cluster-level covariate and the within-cluster covariate. 


\subsubsection{Non-Gaussian random effects}

We consider a gamma random intercept, such that

$$
u_{i j}=u_{i 0}=\sigma\left(a_{i}-\lambda\right) / \sqrt{\lambda}
$$

where

$$
a_{i} \sim \operatorname{gamma}(\lambda, 1), \text { and } \operatorname{var}\left(a_{i}\right)=E(\lambda)
$$

It is easy to show that

$$
E\left(u_{i 0}\right)=\frac{\sigma}{\sqrt{\lambda}} E\left(a_{i}-\lambda\right)=0
$$

and

$$
\begin{aligned}
\operatorname{var}\left(u_{i 0}\right) & =\frac{\sigma^{2}}{\lambda} \operatorname{var}\left(a_{i}-\lambda\right) \\
& =\frac{\sigma^{2}}{\lambda} \operatorname{var}\left(a_{i}\right)=\sigma^{2} .
\end{aligned}
$$

Here it is clear that the random intercept $u_{i 0}$ has mean 0 variance $\sigma^{2}$, but follows a non-normal distribution.

\subsubsection{Heterogeneity dependent on a cluster-level covariate}

Heagerty and Kurland (2001) investigate the asymptotic bias of the ML estimates for using the misspecified Gaussian random intercepts for GLMM's. Recall the regression model

$$
\mathrm{g}\left(\mu_{i j}\right)=\beta_{0}+\beta_{1} X_{i j, 1}+\beta_{2} X_{i j, 2}+\beta_{3} X_{i j, 1} X_{i j, 2}+u_{i j}
$$

Here we assume that variance of the random effects $u_{i j}=u_{i 0}$ depends on the between-cluster covariate $X_{i j, 1}$, that is, 


$$
\left\lfloor u_{i 0} \mid X_{i j, 1}=0\right\rfloor \sim N\left(0, \sigma_{0}^{2}\right)
$$

and

$$
\left\lfloor u_{i 0} \mid X_{i j, 1}=1\right] \sim N\left(0, \sigma_{1}^{2}\right) .
$$

For the special case $\sigma_{0}^{2}=\sigma_{1}^{2}$, the model becomes the ordinary Gaussian random intercepts model.

\subsubsection{Random coefficients}

Recall the regression model

$$
\mathrm{g}\left(\mu_{i j}\right)=\beta_{0}+\beta_{1} X_{i j, 1}+\beta_{2} X_{i j, 2}+\beta_{3} X_{i j, 1} X_{i j, 2}+u_{i j}
$$

where $u_{i j}$ is assumed to have the form

$$
u_{i j}=u_{i, 0}+u_{i, 1} x_{j}
$$

with

$$
u_{i, 0} \sim N\left(0, \sigma_{0}^{2}\right) \text { and } u_{i, 1} \sim N\left(0, \sigma_{1}^{2}\right)
$$

$u_{i, 0}$ and $u_{i, 1}$ are mutually independent. In this case,

$$
\operatorname{var}\left(u_{i j}\right)=\sigma_{0}^{2}+\sigma_{1}^{2} x_{j}^{2}
$$

So for a non-zero value of $x_{j}, \operatorname{var}\left(u_{i j}\right)$ depends on the within-cluster covariate. 


\subsubsection{Autoregressive random effects}

Clusters that arise as a result of repeated measures in a longitudinal study frequently have dependence that decays as the time separation between measurements increases. One model that admits a serial covariance structure is the generalized linear mixed model that includes autocorrelated random effects, $u_{i j}$, for which

$$
\operatorname{cov}\left(u_{i j}, u_{i k}\right)=\sigma^{2} \rho^{|j-k|}
$$

In this case, if we choose $n_{i}=5$, then

$$
u_{i}=\left(u_{i, 1}, \cdots, u_{i, 5}\right) \sim N_{5}(0, \Sigma)
$$

where

$$
\Sigma=\left(\begin{array}{lllll}
1 & \rho & \rho^{2} & \rho^{3} & \rho^{4} \\
\rho & 1 & \rho & \rho^{2} & \rho^{3} \\
\rho^{2} & \rho & 1 & \rho & \rho^{2} \\
\rho^{3} & \rho^{2} & \rho & 1 & \rho \\
\rho^{4} & \rho^{3} & \rho^{2} & \rho & 1
\end{array}\right)
$$

In the next chapter, we carry out a simulation study using these random effects distributions for the GLMM's and investigate the-impact of model violations on the parameter estimates. 


\section{Chapter 5}

\section{Simulation Study}

We ran a series of simulations to investigate the effects of model misspecification when fitting generalized linear mixed models. In particular, we are interested in situations where the relevant regression model, $\mathrm{g}\left(\mu_{i j}\right)$, is correctly specified, but where the distributional assumptions for the random effects, $f\left(u_{i j}\right)$, are misspecified. We compare the widely used ML method to the approximate $\mathrm{PQL}$ method in the simulation study under various types of misspecified models. Details about the simulation results are given below.

\subsection{Non-Gaussian random effects}

Recall the binary mixed model with mean response $\mu_{i j}$ :

$$
\begin{array}{r}
\mathrm{g}\left(\mu_{i j}\right)=\operatorname{logit}\left(\mu_{i j}\right)=\beta_{0}+\beta_{1} X_{i j, 1}+\beta_{2} X_{i j, 2}+\beta_{3} X_{i j, 1} X_{i j, 2}+u_{i j} \\
(\mathrm{i}=1,2, \ldots \mathrm{k} ; \mathrm{j}=1,2, \ldots \mathrm{n})
\end{array}
$$

where $u_{i j}=u_{i, 0}=\sigma\left(a_{i}-\lambda\right) / \sqrt{\lambda}$ with $a_{i} \sim \operatorname{gamma}(\lambda, 1)$. We explore a range of values for the shape parameter $\lambda$ and the scale parameter $\sigma$.

In all cases, the regression parameter $\beta$ is fixed at $\beta=(-2,2,2,1)$. For each set of parameter values, we generate a series of 500 data sets, each with sample 
sizes $\mathrm{k}=40$ or 200 and $\mathrm{n}=5$. Table 1.1 presents the simulated biases for $\mathrm{ML}$ and PQL estimates obtained under the assumption of misspecified Gaussian intercept term for generalized linear mixed models. Table 1.2 presents simulated root mean squared errors of the $\mathrm{ML}$ and $\mathrm{PQL}$ estimates for the misspecified generalized linear mixed models.

The bias of an estimate $\hat{\theta}$ is given by

$$
\begin{aligned}
\operatorname{Bias}(\hat{\theta}) & =\mathrm{E}(\hat{\theta})-\theta \\
& \cong \frac{1}{500} \sum_{s=1}^{500} \hat{\theta}^{(s)}-\theta
\end{aligned}
$$

where $\hat{\theta}^{(s)},(s=1,2,3, \ldots, 500)$ are the parameter estimates obtained from the $s^{\text {th }}$ sample. Also the mean squared error (MSE) of $\hat{\theta}$ is given by

$$
\begin{aligned}
& \operatorname{MSE}(\hat{\theta})=\mathrm{E}(\hat{\theta}-\theta)^{2} \\
& \cong \frac{1}{500} \sum_{s=1}^{500}\left(\hat{\theta}^{(s)}-\theta\right)^{2} .
\end{aligned}
$$

The statistical package $R$ was used for generating the data and for fitting GLMMs to the data by both ML and PQL methods. The R function "glmmML" fits generalized linear mixed models with random intercept by maximum likelihood and numerical integration via Gauss-Hermite quadrature. The function "glmmPQL" fits a generalized linear mixed model -with multivariate normal random effects, using penalized quasi-likelihood. 
Table 1.1:

Simulated biases for ML and PQL estimates under misspecified Gaussian random effects for GLMM's where the random effects have a gamma distribution, $u_{i j}=u_{i, 0}=\sigma\left(a_{i}-\lambda\right) / \sqrt{\lambda}$ for $a_{i} \sim \operatorname{gamma}(\lambda, 1)$.

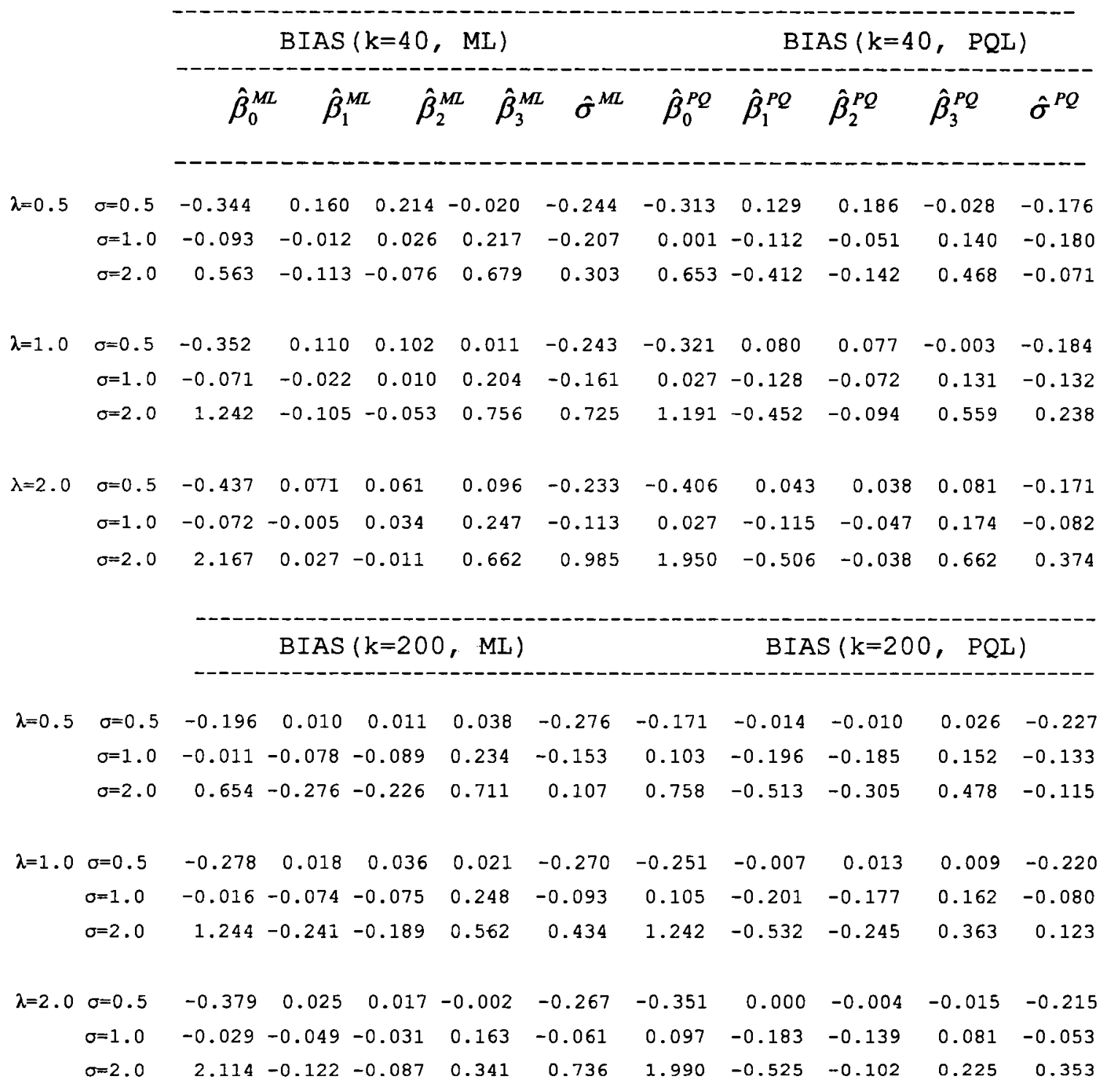


Table 1.2:

Simulated root mean squared errors for ML and PQL estimates under misspecified Gaussian random effects for GLMM's where the random effects have a gamma distribution, $u_{i j}=u_{i, 0}=\sigma\left(a_{i}-\lambda\right) / \sqrt{\lambda}$ for $a_{i} \sim \operatorname{gamma}(\lambda, 1)$.

\begin{tabular}{|c|c|c|c|c|c|c|c|c|c|c|c|}
\hline & \multicolumn{6}{|c|}{$\operatorname{RootMSE}(k=40, \mathrm{ML})$} & \multicolumn{4}{|c|}{$\operatorname{RootMSE}(k=40, \quad P Q I)$} \\
\hline & & $\hat{\beta}_{0}^{M L}$ & $\hat{\beta}_{1}^{M L}$ & $\hat{\beta}_{2}^{M L}$ & $\hat{\beta}_{3}^{M L}$ & $\hat{\sigma}^{M L}$ & $\hat{\beta}_{0}^{P Q}$ & $\hat{\beta}_{1}^{P Q}$ & $\hat{\beta}_{2}^{P Q}$ & $\hat{\beta}_{3}^{P Q}$ & $\hat{\sigma}^{P Q}$ \\
\hline \multirow[t]{3}{*}{$\lambda=0.5$} & $\sigma=0.5$ & 0.663 & 0.704 & 0.811 & 1.229 & 0.389 & 0.638 & 0.685 & 0.790 & 1.221 & 0.374 \\
\hline & $\sigma=1.0$ & 0.611 & 0.739 & 0.775 & 1.223 & 0.462 & 0.591 & 0.719 & 0.758 & 1.156 & 0.430 \\
\hline & $\sigma=2.0$ & 1.128 & 1.358 & 0.870 & 1.556 & 1.432 & 0.993 & 1.047 & 0.868 & 1.516 & 0.491 \\
\hline \multirow[t]{3}{*}{$\lambda=1.0$} & $\sigma=0.5$ & 0.709 & 0.741 & 0.879 & 1.259 & 0.388 & 0.686 & 0.730 & 0.867 & 1.247 & 0.379 \\
\hline & $\sigma=1.0$ & 0.593 & $0.729 \quad 0$ & 0.751 & 1.204 & 0.418 & 0.576 & 0.709 & 0.731 & 1.128 & 0.388 \\
\hline & $\sigma=2.0$ & 1.592 & $1.358 \quad 0$ & 0.990 & 1.887 & 1.290 & 1.451 & 1.150 & 1.016 & 1.734 & 0.543 \\
\hline \multirow[t]{4}{*}{$\lambda=2.0$} & $\sigma=0.5$ & 0.716 & 0.717 & 0.794 & 1.168 & 0.387 & 0.695 & 0.708 & 0.787 & 1.155 & 0.382 \\
\hline & $\sigma=1.0$ & 0.618 & $0.783 \quad 0$ & 0.756 & 1.258 & 0.401 & 0.609 & 0.764 & 0.745 & 1.192 & 0.376 \\
\hline & $\sigma=2.0$ & 2.447 & $1.725 \quad 0$ & 0.963 & 2.346 & 1.774 & 2.110 & 1.204 & 0.945 & 2.639 & 0.581 \\
\hline & & & RootMs & $\mathrm{SE}(\mathrm{k}=$ & $200, \mathrm{M}$ & IL ) & & RootMS & $E(k=2$ & $00, \mathrm{PQ}$ & QI) \\
\hline \multirow[t]{3}{*}{$\lambda=0.5$} & $\sigma=0.5$ & 0.308 & 0.299 & 0.325 & 0.502 & 0.338 & 0.291 & 0.296 & 0.322 & 0.495 & 0.320 \\
\hline & $\sigma=1.0$ & 0.247 & 0.326 & 0.338 & 0.582 & 0.225 & 0.259 & 0.360 & 0.362 & 0.522 & 0.197 \\
\hline & $\sigma=2.0$ & 0.739 & 0.537 & 0.416 & 0.925 & 0.242 & 0.818 & 0.652 & 0.458 & 0.724 & 0.209 \\
\hline \multirow[t]{3}{*}{$\lambda=1.0$} & $\sigma=0.5$ & 0.370 & 0.290 & 0.332 & 0.471 & 0.338 & 0.346 & 0.286 & 0.324 & 0.465 & 0.320 \\
\hline & $\sigma=1.0$ & 0.243 & 0.321 & 0.334 & 0.575 & 0.188 & 0.255 & 0.356 & 0.356 & 0.512 & 0.165 \\
\hline & $\sigma=2.0$ & 1.293 & 0.553 & 0.415 & 0.832 & 0.511 & 1.281 & 0.683 & 0.440 & 0.675 & 0.226 \\
\hline \multirow[t]{3}{*}{$\lambda=2.0$} & $\sigma=0.5$ & 0.456 & 0.306 & 0.348 & 0.507 & 0.331 & 0.430 & 0.299 & 0.342 & 0.501 & 0.313 \\
\hline & $\sigma=1.0$ & 0.251 & 0.335 & 0.322 & 0.551 & 0.168 & 0.257 & 0.361 & 0.335 & 0.498 & 0.147 \\
\hline & $\sigma=2.0$ & 2.147 & 0.610 & 0.408 & 0.759 & 0.807 & 2.016 & 0.706 & 0.411 & 0.689 & 0.408 \\
\hline
\end{tabular}


As seen in Table 1.1 , both ML and PQL methods provide biased estimates under misspecified random effects distribution. The biases appear to increase or decrease monotonically when the values of the shape parameter $\lambda$ and the scale parameter $\sigma$ increase. For example, the biases of both the ML and PQL estimates of $\beta_{0}$ increase when the value of $\sigma$ increases for any fixed value of $\lambda$. Also for any fixed value of $\sigma$, the biases of the ML and PQL estimates of $\beta_{0}$ increase when the value of the shape parameter $\lambda$ increases. The biases of $\hat{\beta}_{0}$ are the smallest for $\sigma=1$.

It is important to note that the PQL method provides smaller biases for estimating $\sigma$ as compared to the ML method for both $k=40$ and $k=200$. But for estimating the regression coefficients, there is no clear indication that $P Q L$ method performs better than the ML method or vice-versa.

From Table 1.2, we observe that the root mean squared errors of both regression coefficients and variance components increase when the values of $\lambda$ and $\sigma$ increase. The PQL method provides uniformly smaller root mean squared errors for the estimates of $\sigma$ as compared to the ML method for all situations considered. As expected, both ML and PQL methods provide smaller root MSE for increased value of $k$, that is, when $k=200$.

\subsection{Heterogeneity dependent on a binary cluster- level covariate}

Here we investigate the situation where the variance component $\sigma^{2}$ depends on the cluster-level covariate. We calculate the biases and root means squared errors

of estimates when a simple random intercepts model for GLMM is fitted assuming

$u_{i j}=u_{i, 0}$ is $N\left(0, \sigma^{2}\right)$, where in fact the data were generated from the mean response. 
Table 2.1:

Simulated biases for ML and PQL estimates under misspecified Gaussian random effects for GLMM's where the variance of the random effects depends on a between-cluster covariate: $\quad\left[u_{i, 0} \mid X_{i j, 1}=0\right] \sim N\left(0, \sigma_{0}{ }^{2}\right)$ and $\left[u_{i, 0} \mid X_{i j, 1}=1\right] \sim N\left(0, \sigma_{1}^{2}\right)$ for $\mathbf{k}=40$.

\begin{tabular}{|c|c|c|c|c|c|c|c|c|c|}
\hline & & BI & $S(k=$ & , ML) & & & BIAS ( & $k=40$, & $Q L)$ \\
\hline & & $\hat{\beta}_{0}^{M L}$ & $\hat{\beta}_{1}^{M L}$ & $\hat{\beta}_{2}^{M L}$ & $\hat{\beta}_{3}^{M L}$ & $\hat{\beta}_{0}^{P Q}$ & $\hat{\beta}_{1}^{P Q}$ & $\hat{\beta}_{2}^{P Q}$ & $\hat{\beta}_{3}^{P Q}$ \\
\hline$\sigma_{1}=0.5$ & $\sigma_{0}=0.5$ & 0.080 & -0.020 & -0.057 & 0.303 & 0.118 & -0.042 & -0.077 & 0.268 \\
\hline & $\sigma_{0}=1.0$ & -0.015 & -0.435 & 0.034 & 0.607 & -0.084 & -0.514 & 0.058 & 0.497 \\
\hline & $\sigma_{0}=2.0$ & 0.199 & -0.179 & -0.195 & 0.774 & 0.299 & -0.311 & -0.239 & 0.511 \\
\hline & $\sigma_{0}=3.0$ & 0.382 & -0.465 & -0.398 & 1.995 & 0.484 & -0.624 & -0.453 & 1.741 \\
\hline$\sigma_{1}=1.0$ & $\sigma_{0}=0.5$ & -0.430 & 0.275 & 0.564 & -0.604 & -0.321 & 0.156 & 0.458 & -0.600 \\
\hline & $\sigma_{0}=1.0$ & -0.081 & 0.061 & -0.069 & 0.466 & -0.190 & 0.073 & -0.103 & -0.492 \\
\hline & $\sigma_{0}=2.0$ & 0.121 & 0.028 & -0.141 & 0.375 & 0.247 & -0.159 & -0.241 & 0.270 \\
\hline & $\sigma_{0}=3.0$ & 0.758 & -0.601 & -0.706 & 1.000 & 0.843 & -0.781 & -0.746 & 0.722 \\
\hline$\sigma_{1}=2.0$ & $\sigma_{0}=0.5$ & -0.223 & 0.092 & 0.029 & -0.008 & -0.036 & -0.135 & -0.123 & 0.015 \\
\hline & $\sigma_{0}=1.0$ & -0.386 & 0.255 & 0.152 & -0.269 & -0.160 & -0.033 & -0.014 & -0.195 \\
\hline & $\sigma_{0}=2.0$ & 0.229 & -0.175 & -0.113 & -0.330 & 0.382 & -0.398 & -0.236 & -0.382 \\
\hline & $\sigma_{0}=3.0$ & -0.711 & 0.861 & 0.656 & 0.449 & -0.562 & 0.458 & 0.679 & 0.160 \\
\hline$\sigma_{1}=3.0$ & $\sigma_{0}=0.5$ & -0.775 & 0.894 & 0.711 & -1.379 & -0.510 & 0.538 & 0.485 & -1.172 \\
\hline & $\sigma_{0}=1.0$ & -0.636 & 0.884 & 0.363 & -1.049 & -0.325 & 0.422 & 0.166 & -0.847 \\
\hline & $\sigma_{0}=2.0$ & -0.635 & 0.273 & 0.367 & -0.182 & -0.109 & -0.272 & 0.195 & -0.198 \\
\hline & $\sigma_{0}=3.0$ & 0.075 & -0.045 & -0.107 & -0.343 & 0.180 & -0.167 & -0.124 & -0.352 \\
\hline
\end{tabular}


Table 2.2:

Simulated biases for ML and PQL estimates under missspecified Gaussian random effects for GLMM's where the variance of the random effects depends on a between-cluster covariate: $\quad\left[u_{i, 0} \mid X_{i j, 1}=0\right] \sim N\left(0, \sigma_{0}{ }^{2}\right) \quad$ and $\left[u_{i, 0} \mid X_{i j, 1}=1\right] \sim N\left(0, \sigma_{1}^{2}\right)$ for $\mathrm{k}=200$

\begin{tabular}{|c|c|c|c|c|c|c|c|c|c|}
\hline & & BI & $\mathrm{AS}(\mathrm{k}=20$ & $0, \quad M L)$ & & & BIAS & $(k=200$ & PQL) \\
\hline & & $\hat{\beta}_{0}^{M L}$ & $\hat{\beta}_{1}^{M L}$ & $\hat{\beta}_{2}^{M L}$ & $\hat{\beta}_{3}^{M L}$ & $\hat{\beta}_{0}^{P Q}$ & $\hat{\beta}_{1}^{P Q}$ & $\hat{\beta}_{2}^{P Q}$ & $\hat{\beta}_{3}^{P Q}$ \\
\hline$\sigma_{1}=0.5$ & $\sigma_{0}=0.5$ & 0.047 & -0.009 & -0.043 & -0.021 & 0.098 & -0.028 & -0.106 & -0.041 \\
\hline & $\sigma_{0}=1.0$ & -0.091 & 0.084 & -0.177 & 0.305 & -0.096 & 0.133 & -0.286 & 0.302 \\
\hline & $\sigma_{0}=2.0$ & 0.254 & -0.144 & -0.272 & 0.798 & 0.374 & -0.301 & -0.358 & 0.582 \\
\hline & $\sigma_{0}=3.0$ & 0.435 & -0.239 & -0.375 & 0.853 & 0.565 & -0.435 & -0.456 & 0.564 \\
\hline$\sigma_{1}=1.0$ & $\sigma_{0}=0.5$ & -0.085 & 0.067 & 0.150 & -0.226 & 0.028 & -0.051 & 0.042 & -0.247 \\
\hline & $\sigma_{0}=1.0$ & -0.021 & 0.047 & -0.014 & 0.130 & -0.123 & -0.490 & -0.124 & 0.190 \\
\hline & $\sigma_{0}=2.0$ & 0.234 & -0.181 & -0.232 & 0.561 & 0.377 & -0.370 & -0.335 & 0.376 \\
\hline & $\sigma_{0}=3.0$ & 0.500 & -0.471 & -0.227 & 0.653 & 0.619 & -0.645 & -0.309 & 0.395 \\
\hline$\sigma_{1}=2.0$ & $\sigma_{0}=0.5$ & -0.311 & 0.293 & 0.316 & -0.835 & -0.116 & 0.071 & 0.134 & -0.790 \\
\hline & $\sigma_{0}=1.0$ & -0.151 & 0.218 & 0.096 & -0.391 & 0.058 & -0.056 & -0.080 & -0.358 \\
\hline & $\sigma_{0}=2.0$ & 0.141 & 0.067 & -0.070 & -0.109 & 0.227 & -0.218 & -0.207 & -0.098 \\
\hline & $\sigma_{0}=3.0$ & 0.200 & -0.034 & -0.002 & 0.346 & 0.376 & -0.374 & -0.081 & 0.169 \\
\hline$=3.0$ & $\sigma_{0}=0.5$ & -0.530 & 0.362 & 0.497 & -1.171 & -0.253 & 0.039 & 0.249 & -1.036 \\
\hline & $\sigma_{0}=1.0$ & -0.366 & 0.326 & 0.250 & -1.060 & -0.086 & -0.044 & 0.024 & -0.920 \\
\hline & $\sigma_{0}=2.0$ & -0.020 & -0.038 & -0.056 & -0.074 & 0.224 & -0.422 & -0.193 & -0.071 \\
\hline & $\sigma_{0}=3.0$ & 0.014 & -0.010 & -0.056 & 0.068 & 0.123 & -0.086 & -0.102 & -0.341 \\
\hline
\end{tabular}


Table 2.3:

Root mean squared error for $\mathrm{ML}$ and $\mathrm{PQL}$ estimates under misspecified Gaussian random effects for GLMM's where the variance of the random effects depends on a between-cluster covariate: $\left[u_{i, 0} \mid X_{i j, 1}=0\right] \sim N\left(0, \sigma_{0}{ }^{2}\right)$ and $\left[u_{i, 0} \mid X_{i j, 1}=1\right] \sim N\left(0, \sigma_{1}^{2}\right)$ for $\mathrm{k}=40$.

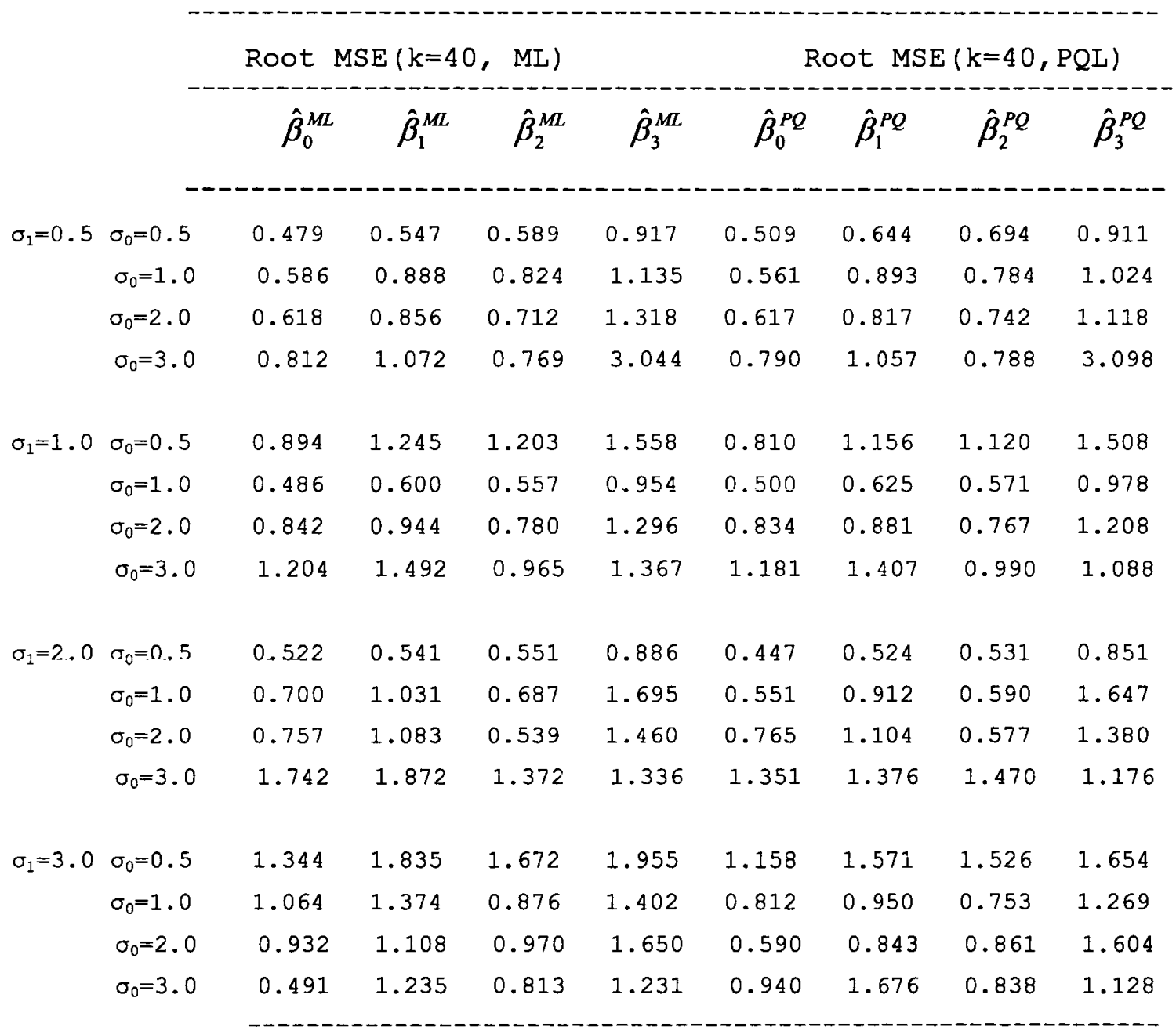


Table 2.4:

Root mean squared error for $\mathrm{ML}$ and $\mathrm{PQL}$ estimates under misspecified Gaussian random effects for GLMM's where the variance of the random effects depends on a between-cluster covariate: $\left[u_{i, 0} \mid X_{i j, 1}=0\right] \sim N\left(0, \sigma_{0}{ }^{2}\right)$ and $\left[u_{i, 0} \mid X_{i j, 1}=1\right] \sim N\left(0, \sigma_{1}^{2}\right)$ for $\mathrm{k}=200$.

\begin{tabular}{|c|c|c|c|c|c|c|c|c|c|}
\hline \multicolumn{6}{|c|}{ Root $\operatorname{MSE}(k=200, M L)$} & \multicolumn{4}{|c|}{ Root $\operatorname{MSE}(k=200, P Q L)$} \\
\hline & & $\hat{\beta}_{0}^{M L}$ & $\hat{\beta}_{1}^{M L}$ & $\hat{\beta}_{2}^{M L}$ & $\hat{\beta}_{3}^{M L}$ & $\hat{\beta}_{0}^{P Q}$ & $\hat{\beta}_{1}^{P Q}$ & $\hat{\beta}_{2}^{P Q}$ & $\hat{\beta}_{3}^{P Q}$ \\
\hline \multirow[t]{4}{*}{$\sigma_{1}=0.5$} & $\sigma_{0}=0.5$ & 0.223 & 0.252 & 0.324 & 0.415 & 0.240 & 0.262 & 0.329 & 0.409 \\
\hline & $\sigma_{0}=1.0$ & 0.195 & 0.277 & 0.252 & 0.548 & 0.211 & 0.343 & 0.336 & 0.531 \\
\hline & $\sigma_{0}=2.0$ & 0.353 & 0.386 & 0.356 & 0.875 & 0.442 & 0.451 & 0.424 & 0.669 \\
\hline & $\sigma_{0}=3.0$ & 0.514 & 0.480 & 0.544 & 0.984 & 0.624 & 0.580 & 0.600 & 0.735 \\
\hline \multirow[t]{4}{*}{$\sigma_{1}=1.0$} & $\sigma_{0}=0.5$ & 0.190 & 0.225 & 0.335 & 0.502 & 0.161 & 0.208 & 0.281 & 0.492 \\
\hline & $\sigma_{0}=1.0$ & 0.080 & 0.249 & 0.208 & 0.356 & 0.144 & 0.303 & 0.242 & 0.357 \\
\hline & $\sigma_{0}=2.0$ & 0.345 & 0.495 & 0.330 & 0.786 & 0.444 & 0.556 & 0.401 & 0.624 \\
\hline & $\sigma_{0}=3.0$ & 0.591 & 0.666 & 0.340 & 0.803 & 0.683 & 0.772 & 0.412 & 0.585 \\
\hline \multirow[t]{4}{*}{$\sigma_{1}=2.0$} & $\sigma_{0}=0.5$ & 0.390 & 0.512 & 0.498 & 1.084 & 0.244 & 0.391 & 0.375 & 1.017 \\
\hline & $\sigma_{0}=1.0$ & 0.341 & 0.330 & 0.471 & 0.673 & 0.281 & 0.210 & 0.426 & 0.631 \\
\hline & $\sigma_{0}=2.0$ & 0.260 & 0.344 & 0.245 & 0.458 & 0.382 & 0.398 & 0.305 & 0.480 \\
\hline & $\sigma_{0}=3.0$ & 0.485 & 0.652 & 0.381 & 0.600 & 0.549 & 0.649 & 0.377 & 0.483 \\
\hline \multirow[t]{4}{*}{$\sigma_{1}=3.0$} & $\sigma_{0}=0.5$ & 0.655 & 0.515 & 0.652 & 1.229 & 0.426 & 0.323 & 0.456 & 1.087 \\
\hline & $\sigma_{0}=1.0$ & 0.468 & 0.559 & 0.479 & 1.151 & 0.276 & 0.383 & 0.365 & 1.012 \\
\hline & $\sigma_{0}=2.0$ & 0.439 & 0.627 & 0.724 & 0.852 & 0.465 & 0.709 & 0.702 & 0.773 \\
\hline & $\sigma_{0}=3.0$ & 0.432 & 0.841 & 0.401 & 0.590 & 0.470 & 0.832 & 0.406 & 0.550 \\
\hline
\end{tabular}




$$
g\left(\mu_{i j}\right)=\log i t\left(\mu_{i j}\right)=\beta_{0}+\beta_{1} X_{i j, 1}+\beta_{2} X_{i j, 2}+\beta_{3} X_{i j, 1} X_{i j, 2}+u_{i j}
$$

$$
(i=1,2,3, \ldots ., k ; j=1,2,3, \ldots . ., n)
$$

with $u_{i j}=u_{i, 0}$ and $\left[u_{i, 0} \mid X_{i j, 1}=0\right] \sim N\left(0, \sigma_{0}^{2}\right),\left[u_{i j} \mid X_{i j, 1}=1\right] \sim N\left(0, \sigma_{1}^{2}\right), \sigma_{0}^{2} \neq \sigma_{1}^{2}$.

Note that for the choice $\sigma_{0}^{2}=\sigma_{1}^{2}=\sigma^{2}$, we have $\left[u_{i, 0} \mid x_{i}\right] \sim N\left(0, \sigma^{2}\right)$ and there is no violation of model assumptions.

As before, we explore a range values for $\sigma_{0}$ and $\sigma_{1}$, with the values of $\beta$ coefficients being fixed at $\beta=(-2,2,2,1)$. For each set of parameter values, a series of 500 data sets are generated with sample sizes $k=(40,200)$ and $n=5$.

Tables 2.1 and 2.2 present the simulated biases of parameter estimates for $k=40$ and $k=200$, respectively. As expected, we observe that when there is no violation of model assumptions (that is, when $\sigma_{0}=\sigma_{1}$ ), the ML method provides smaller biases as compared to the PQL method for all regression coefficients. Substantial bias occurs for all coefficients when $\sigma_{0}$ is far from $\sigma_{1}$. For example, in Table 2.2, when $\sigma_{0}=2.0$ and $\sigma_{1}=1.0$, but $\sigma_{0}=\sigma_{1}$ is assumed, the $\mathrm{ML}$ method provides the biases $(0.234,-0.181,-0.232,0.561)$ for $\left(\hat{\beta}_{0}, \hat{\beta}_{1}, \hat{\beta}_{2}, \hat{\beta}_{3}\right)$ and the PQL method provides corresponding biases as $(0.377,-0.370,-0.335$, 0.376). The biases decrease to some extent for increased value of the sample size $-\mathrm{k}$. Also observe that the PQL method generally provides smaller biases for $\hat{\beta}_{3}$ as compared to the ML method when $\sigma_{0} \neq \sigma_{1}$.

Tables 2.3 and 2.4 present the simulated root mean squared errors of the ML and PQL estimates for sample sizes $k=40$ and $k=200$, respectively. Here also we observe that for correctly specified model $\left(\sigma_{0}=\sigma_{1}\right)$, the ML method provides the smallest root MSEs. When $\sigma_{0}$ is far from $\sigma_{1}$, in many cases PQL method appears 
to be more efficient than the ML method. For example, when $\sigma_{0}=1$ and $\sigma_{1}=3$, the PQL method provides the root MSEs $(0.812,0.950,0.753,1.269)$ for $\left(\hat{\beta}_{0}, \hat{\beta}_{1}\right.$, $\left.\hat{\beta}_{2}, \hat{\beta}_{3}\right)$ and the ML method provides the corresponding $(1.064,1.374,0.876$, 1.402) as seen in Table 2.3 for $\mathbf{k}=40$. The root MSEs are reduced when $\mathbf{k}=200$ in all situations, as expected.

\subsection{Random intercepts and random slopes are mutually independent.}

Longford (1993) use a popular formulation for generalized linear mixed models in terms of fixed and random coefficients (Longford, 1993). This model for conditionally specified mean response is

$$
g\left(\mu_{i j}\right)=\beta_{0}+\beta_{1} X_{i j, 1}+\beta_{2} X_{i j, 2}+\beta_{3} X_{i j, 1} X_{i j, 2}+\left(u_{i, 0}+u_{i, 1} X_{i j, 2}\right)
$$

where

$$
u_{i, 0} \sim N\left(0, \sigma_{0}^{2}\right) \text { and } u_{i, 1} \sim N\left(0, \sigma_{1}^{2}\right) \text { are mutually independent. }
$$

Here we investigate a range of values for $\sigma_{0}$ and $\sigma_{1}$. In each case, the regression coefficients in $\beta$ were fixed at $\beta=(-2,2,2,1)$. As before, a series of 500 data sets were generated for each set of parameter values.

Tables 3.1 and 3.2 present the simulated biases of the estimates of regression coefficients when a simple random intercepts model for the GLMM is fitted to data assuming that $u_{i j}=u_{i, 0} \sim N\left(0, \sigma^{2}\right)$, where in fact $u_{i j}=u_{i, 0}+u_{i, 1} x_{j} \sim$ $N\left(0, \sigma_{0}^{2}+\sigma_{1}^{2} x_{j}^{2}\right)$. In both small sample $(\mathrm{k}=40)$ and large sample $(\mathrm{k}=200)$ cases, we observe that when $\sigma_{1}$ is fixed at small values, the PQL method provides larger biases for larger values of $\sigma_{0}$ as compared to the ML method. 


\section{Table 3.1:}

Simulated biases for ML and PQL estimates under misspecified Gaussian random effects for GLMM's, when there are mutually independent random intercepts and random slopes: $u_{i j}=u_{i, 0}+u_{i, 1} x_{j}$ with $u_{i, 0} \sim N\left(0, \sigma_{0}^{2}\right)$ and $u_{i 1} \sim N\left(0, \sigma_{1}^{2}\right)$ for $\mathrm{k}=40$.

\begin{tabular}{|c|c|c|c|c|c|c|c|c|c|}
\hline & \multicolumn{4}{|c|}{$\operatorname{BIAS}(k=40, \mathrm{ML})$} & & \multicolumn{3}{|c|}{$\operatorname{BIAS}(k=40, \quad P Q L)$} \\
\hline & & $\hat{\beta}_{0}^{M L}$ & $\hat{\beta}_{1}^{M L}$ & $\hat{\beta}_{2}^{M L}$ & $\hat{\beta}_{3}^{M L}$ & $\hat{\beta}_{0}^{P Q}$ & $\hat{\beta}_{1}^{P Q}$ & $\hat{\beta}_{2}^{P Q}$ & $\hat{\beta}_{3}^{P Q}$ \\
\hline \multirow[t]{4}{*}{$\sigma_{1}=0.2$} & $\sigma_{0}=0.5$ & -0.239 & 0.271 & 0.428 & -0.602 & -0.164 & 0.189 & 0.347 & -0.617 \\
\hline & $\sigma_{0}=1.0$ & 0.507 & -0.610 & -0.607 & 0.562 & 0.581 & -0.693 & -0.668 & 0.485 \\
\hline & $\sigma_{0}=2.0$ & 0.028 & -0.102 & 0.238 & -0.326 & 0.211 & -0.352 & 0.072 & -0.362 \\
\hline & $\sigma_{0}=3.0$ & 0.103 & -0.345 & -0.099 & -0.050 & 0.304 & -0.668 & -0.210 & -0.124 \\
\hline \multirow[t]{4}{*}{$\sigma_{1}=0.5$} & $\sigma_{0}=0.5$ & -0.045 & 0.233 & -0.038 & -0.441 & 0.025 & 0.153 & -0.104 & -0.444 \\
\hline & $\sigma_{0}=1.0$ & -0.083 & 0.299 & 0.309 & -0.266 & -0.213 & 0.483 & 0.432 & -0.222 \\
\hline & $\sigma_{0}=2.0$ & 0.177 & -0.406 & -0.139 & 0.130 & 0.353 & -0.637 & -0.265 & 0.062 \\
\hline & $\sigma_{0}=3.0$ & -0.249 & 0.316 & -0.392 & 0.812 & 0.346 & -0.393 & -0.446 & 0.670 \\
\hline \multirow[t]{4}{*}{$\sigma_{1}=0.8$} & $\sigma_{0}=0.5$ & -0.027 & 0.066 & 0.014 & 0.468 & 0.020 & 0.007 & -0.027 & 0.515 \\
\hline & $\sigma_{0}=1.0$ & 0.058 & -0.225 & -0.192 & 0.310 & 0.178 & -0.362 & -0.288 & 0.206 \\
\hline & $\sigma_{0}=2.0$ & -0.246 & 0.192 & -0.219 & 0.661 & 0.058 & -0.215 & -0.347 & 0.540 \\
\hline & $\sigma_{0}=3.0$ & -0.482 & -0.347 & 0.938 & 0.065 & -0.187 & -0.968 & 0.734 & 0.193 \\
\hline \multirow[t]{4}{*}{$\sigma_{1}=1.0$} & $\sigma_{0}=0.5$ & 0.174 & -0.654 & -0.347 & 0.910 & 0.257 & -0.713 & -0.424 & 0.839 \\
\hline & $\sigma_{0}=1.0$ & -0.343 & 0.634 & 0.280 & -0.618 & -0.198 & 0.441 & 0.170 & -0.619 \\
\hline & $\sigma_{0}=2.0$ & -0.570 & 1.251 & 0.317 & -0.872 & -0.343 & 0.825 & 0.241 & -0.941 \\
\hline & $\sigma_{0}=3.0$ & 0.514 & -0.994 & 0.090 & 0.433 & 0.649 & -1.040 & 0.240 & 0.399 \\
\hline
\end{tabular}




\section{Table 3.2:}

Simulated biases for ML and PQL estimates under misspecified Gaussian random effects for GLMM's, when there are mutually independent random intercepts and random slopes: $u_{i j}=u_{i, 0}+u_{i, 1} x_{j}$ with $u_{i, 0} \sim N\left(0, \sigma_{0}^{2}\right)$ and $u_{i, 1} \sim N\left(0, \sigma_{1}^{2}\right)$ for $\mathrm{k}=200$.

\begin{tabular}{|c|c|c|c|c|c|c|c|c|c|}
\hline \multirow{2}{*}{\multicolumn{2}{|c|}{-}} & \multicolumn{5}{|c|}{$\operatorname{BIAS}(k=200, M L)$} & \multicolumn{3}{|c|}{$\operatorname{BIAS}(k=200, \quad P Q L)$} \\
\hline & & $\hat{\beta}_{0}^{M L}$ & $\hat{\beta}_{1}^{M L}$ & $\hat{\beta}_{2}^{M L}$ & $\hat{\beta}_{3}^{M L}$ & $\hat{\beta}_{0}^{P Q}$ & $\hat{\beta}_{1}^{P Q}$ & $\hat{\beta}_{2}^{P Q}$ & $\hat{\beta}_{3}^{P Q}$ \\
\hline \multirow[t]{4}{*}{$\sigma_{1}=0.2$} & $\sigma_{0}=0.5$ & -0.181 & 0.250 & 0.209 & -0.098 & -0.124 & 0.188 & 0.154 & -0.117 \\
\hline & $\sigma_{0}=1.0$ & 0.012 & 0.081 & 0.092 & -0.109 & 0.081 & 0.001 & 0.024 & -0.139 \\
\hline & $\sigma_{0}=2.0$ & 0.099 & -0.080 & -0.126 & 0.200 & 0.295 & -0.363 & -0.262 & 0.105 \\
\hline & $\sigma_{0}=3.0$ & 0.268 & -0.376 & -0.194 & 0.375 & 0.241 & -0.262 & -0.173 & -0.059 \\
\hline \multirow[t]{4}{*}{$\sigma_{1}=0.5$} & $\sigma_{0}=0.5$ & 0.010 & -0.027 & 0.255 & -0.331 & 0.191 & -0.425 & 0.131 & -0.307 \\
\hline & $\sigma_{0}=1.0$ & 0.027 & -0.189 & -0.008 & -0.033 & 0.094 & -0.254 & -0.073 & -0.063 \\
\hline & $\sigma_{0}=2.0$ & 0.087 & -0.220 & -0.089 & 0.260 & 0.211 & -0.349 & -0.202 & 0.185 \\
\hline & $\sigma_{0}=3.0$ & -0.221 & 0.231 & 0.353 & -0.470 & -0.008 & -0.060 & 0.187 & -0.507 \\
\hline \multirow[t]{4}{*}{$\sigma_{1}=0.8$} & $\sigma_{0}=0.5$ & 0.047 & -0.226 & -0.075 & 0.109 & 0.250 & -0.599 & -0.174 & 0.016 \\
\hline & $\sigma_{0}=1.0$ & -0.067 & 0.133 & 0.096 & -0.296 & 0.026 & 0.029 & 0.004 & -0.319 \\
\hline & $\sigma_{0}=2.0$ & -0.075 & 0.119 & 0.103 & -0.246 & 0.069 & -0.046 & -0.028 & -0.291 \\
\hline & $\sigma_{0}=3.0$ & 0.247 & -0.106 & -0.220 & 0.092 & 0.429 & -0.382 & -0.348 & 0.002 \\
\hline \multirow[t]{4}{*}{$\sigma_{1}=1.0$} & $\sigma_{0}=0.5$ & -0.183 & 0.402 & 0.088 & -0.809 & 0.075 & -0.060 & -0.036 & -0.801 \\
\hline & $\sigma_{0}=1.0$ & 0.061 & 0.009 & -0.227 & 0.172 & 0.151 & -0.094 & -0.304 & 0.124 \\
\hline & $\sigma_{0}=2.0$ & -0.048 & 0.105 & 0.148 & -0.560 & 0.103 & -0.073 & 0.004 & -0.584 \\
\hline & $\sigma_{0}=3.0$ & -0.062 & -0.013 & 0.101 & -0.405 & 0.252 & -0.448 & 0.244 & -0.340 \\
\hline
\end{tabular}




\section{Table 3.3:}

Root mean squared errors for ML and PQL estimates under misspecified Gaussian random effects for GLMM's, when there are mutually independent random intercepts and random slopes: $u_{i j}=u_{i, 0}+u_{i, 1} x_{j}$ with $u_{i, 0} \sim N\left(0, \sigma_{0}^{2}\right)$ and $u_{i, 1} \sim N\left(0, \sigma_{1}^{2}\right)$ for $\mathrm{k}=40$.

\begin{tabular}{|c|c|c|c|c|c|c|c|c|c|}
\hline & & \multicolumn{4}{|c|}{$\operatorname{Root} \operatorname{MSE}(k=40, M L)$} & \multicolumn{4}{|c|}{ Root $\operatorname{MSE}(k=40, \mathrm{PQL})$} \\
\hline & & $\hat{\beta}_{0}^{M L}$ & $\hat{\beta}_{1}^{M L}$ & $\hat{\beta}_{2}^{M L}$ & $\hat{\beta}_{3}^{M L}$ & $\hat{\beta}_{0}^{P Q}$ & $\hat{\beta}_{1}^{P Q}$ & $\hat{\beta}_{2}^{P Q}$ & $\hat{\beta}_{3}^{P Q}$ \\
\hline \multirow[t]{4}{*}{$\sigma_{1}=0.2$} & $\sigma_{0}=0.5$ & 0.568 & 0.552 & 0.847 & 0.920 & 0.496 & 0.462 & 0.746 & 0.895 \\
\hline & $\sigma_{0}=1.0$ & 0.711 & 0.873 & 0.723 & 0.755 & 0.741 & 0.893 & 0.760 & 0.683 \\
\hline & $\sigma_{0}=2.0$ & 0.747 & 1.230 & 0.788 & 0.999 & 0.709 & 1.126 & 0.702 & 0.918 \\
\hline & $\sigma_{0}=3.0$ & 0.929 & 1.478 & 0.981 & 1.314 & 0.836 & 1.322 & 0.953 & 1.196 \\
\hline \multirow[t]{4}{*}{$\sigma_{1}=0.5$} & $\sigma_{0}=0.5$ & 0.485 & 0.377 & 0.547 & 0.938 & 0.454 & 0.317 & 0.523 & 0.930 \\
\hline & $\sigma_{0}=1.0$ & 0.444 & 0.809 & 0.824 & 1.766 & 0.531 & 0.972 & 0.938 & 1.866 \\
\hline & $\sigma_{0}=2.0$ & 0.720 & 0.855 & 0.996 & 0.511 & 0.777 & 0.943 & 0.987 & 0.463 \\
\hline & $\sigma_{0}=3.0$ & 0.916 & 1.329 & 0.553 & 1.408 & 0.692 & 0.709 & 0.625 & 1.327 \\
\hline \multirow[t]{4}{*}{$\sigma_{1}=0.8$} & $\sigma_{0}=0.5$ & 0.519 & 0.479 & 0.843 & 1.438 & 0.502 & 0.459 & 0.823 & 1.533 \\
\hline & $\sigma_{0}=1.0$ & 0.670 & 0.652 & 0.930 & 1.579 & 0.691 & 0.676 & 0.914 & 1.452 \\
\hline & $\sigma_{0}=2.0$ & 1.092 & 1.784 & 0.784 & 1.405 & 0.902 & 1.352 & 0.772 & 1.385 \\
\hline & $\sigma_{0}=3.0$ & 1.185 & 1.945 & 1.609 & 1.631 & 0.854 & 1.237 & 1.440 & 1.545 \\
\hline \multirow[t]{4}{*}{$\sigma_{1}=1.0$} & $\sigma_{0}=0.5$ & 0.334 & 0.716 & 0.746 & 1.654 & 0.344 & 0.768 & 0.732 & 1.587 \\
\hline & $\sigma_{0}=1.0$ & 0.808 & 1.360 & 0.784 & 1.462 & 0.724 & 1.201 & 0.756 & 1.430 \\
\hline & $\sigma_{0}=2.0$ & 0.900 & 0.784 & 1.005 & 1.238 & 0.953 & 0.811 & 0.969 & 1.227 \\
\hline & $\sigma_{0}=3.0$ & 0.923 & 1.162 & 1.105 & 1.340 & 0.738 & 1.286 & 1.011 & 1.388 \\
\hline
\end{tabular}




\section{Table 3.4:}

Root mean squared errors for $\mathrm{ML}$ and $\mathrm{PQL}$ estimates under misspecified Gaussian random effects for GLMM's, when there are mutually independent random intercepts and random slopes: $u_{i j}=u_{i, 0}+u_{i, 1} x_{j}$ with $u_{i, 0} \sim N\left(0, \sigma_{0}^{2}\right)$ and $u_{i, 1} \sim N\left(0, \sigma_{1}^{2}\right)$ for $\mathrm{k}=200$.

\begin{tabular}{|c|c|c|c|c|c|c|c|c|c|}
\hline & \multicolumn{4}{|c|}{ Root $\operatorname{MSE}(k=200, M L)$} & \multicolumn{4}{|c|}{ Root $\operatorname{MSE}(k=200$, PQL $)$} \\
\hline & & $\hat{\beta}_{0}^{M I}$ & $\hat{\beta}_{1}^{M L}$ & $\hat{\beta}_{2}^{M L}$ & $\hat{\beta}_{3}^{M L}$ & $\hat{\beta}_{0}^{P Q}$ & $\hat{\beta}_{1}^{P Q}$ & $\hat{\beta}_{2}^{P Q}$ & $\hat{\beta}_{3}^{P Q}$ \\
\hline \multirow[t]{4}{*}{$\sigma_{1}=0.2$} & $\sigma_{0}=0.5$ & 0.293 & 0.431 & 0.303 & 0.700 & 0.245 & 0.395 & 0.256 & 0.677 \\
\hline & $\sigma_{0}=1.0$ & 0.227 & 0.442 & 0.382 & 0.495 & 0.232 & 0.414 & 0.365 & 0.480 \\
\hline & $\sigma_{0}=2.0$ & 0.349 & 0.338 & 0.468 & 0.872 & 0.427 & 0.462 & 0.494 & 0.752 \\
\hline & $\sigma_{0}=3.0$ & 0.463 & 0.711 & 0.567 & 1.059 & 0.553 & 0.812 & 0.592 & 0.958 \\
\hline \multirow[t]{4}{*}{$\sigma_{1}=0.5$} & $\sigma_{0}=0.5$ & 0.334 & 0.522 & 0.381 & 0.455 & 0.427 & 0.733 & 0.304 & 0.396 \\
\hline & $\sigma_{0}=1.0$ & 0.239 & 0.331 & 0.367 & 0.284 & 0.255 & 0.371 & 0.367 & 0.272 \\
\hline & $\sigma_{0}=2.0$ & 0.328 & 0.369 & 0.362 & 0.471 & 0.368 & 0.449 & 0.387 & 0.408 \\
\hline & $\sigma_{0}=3.0$ & 0.387 & 0.522 & 0.407 & 0.710 & 0.412 & 0.673 & 0.575 & 0.805 \\
\hline \multirow[t]{4}{*}{$\sigma_{1}=0.8$} & $\sigma_{0}=0.5$ & 0.202 & 0.293 & 0.306 & 0.622 & 0.312 & 0.645 & 0.334 & 0.584 \\
\hline & $\sigma_{0}=1.0$ & 0.317 & 0.413 & 0.474 & 0.707 & 0.296 & 0.376 & 0.436 & 0.696 \\
\hline & $\sigma_{0}=2.0$ & 0.212 & 0.387 & 0.226 & 0.397 & 0.199 & 0.347 & 0.187 & 0.418 \\
\hline & $\sigma_{0}=3.0$ & 0.299 & 0.447 & 0.443 & 0.687 & 0.453 & 0.532 & 0.504 & 0.628 \\
\hline \multirow[t]{4}{*}{$\sigma_{1}=0.8$} & $\sigma_{0}=0.5$ & 0.240 & 0.593 & 0.219 & 0.982 & 0.098 & 0.407 & 0.187 & 0.958 \\
\hline & $\sigma_{0}=1.0$ & 0.187 & 0.232 & 0.358 & 0.285 & 0.228 & 0.239 & 0.404 & 0.250 \\
\hline & $\sigma_{0}=2.0$ & 0.365 & 0.505 & 0.505 & 0.564 & 0.359 & 0.415 & 0.487 & 0.572 \\
\hline & $\sigma_{0}=3.0$ & 0.367 & 0.673 & 0.477 & 0.805 & 0.412 & 0.815 & 0.575 & 0.805 \\
\hline
\end{tabular}


For example, when $\sigma_{0}=3$ and $\sigma_{1}=0.2$, from Table 3.1, the ML method provides biases $(0.103,-0.345,-0.099,-0.050)$ for $\left(\hat{\beta}_{0}, \hat{\beta}_{1}, \hat{\beta}_{2}, \hat{\beta}_{3}\right)$ and the PQL method provides biases $(0.304,-0.668,-0.210,-0.120)$. On the other hands, when $\sigma_{0}=1$ and $\sigma_{1}=1$ in Table 3.1, the PQL method provides smaller biases of $(-0.198$, $0.441,0.170,-0.619)$ as compared to the ML method with biases of $(-0.343$, $0.634,0.280,-0.618)$. In both cases, the biases are generally reduced for larger sample size of $\mathrm{k}=200$.

Tables 3.3 and 3.4 present the simulated root mean squared errors of the ML and $P Q L$ estimates for sample sizes $k=40$ and $k=200$, respectively. Here we observe that when $\sigma_{1}$ is larger than $\sigma_{0}$, the PQL method provides more efficient estimates in some cases. For example, when $\sigma_{1}=1.0$ and $\sigma_{0}=0.5$, Table 3.4 shows biases of $(0.240,0.593,0.219,0.982)$ for the ML method and $(0.098,0.407,0.187,0.958)$ for the PQL method. But when $\sigma_{0}$ is larger than $\sigma_{1}$, in many cases the ML method appears to provide more efficient estimates than the PQL method. In general, we observe that when the extent of model misspecification is small (that is, when $\sigma_{1}$ is small but $\sigma_{0}$ is large), the ML method provides more efficient estimates, as expected. The PQL method generally provides more robust estimates under the violation of model assumptions.

\subsection{Autoregressive Random Effects}

Clusters that arise as a result of repeated measures in a longitudinal study frequently have dependence that decays as the time separation between measurements increases. One model that admits a serial covariance structure is the generalized linear mixed model that includes autocorrelated random effects, $u_{i j}$, for which $\operatorname{cov}\left(u_{i j}, u_{i k}\right)=\sigma^{2} \rho^{|j-k|}$. 
Table 4.1:

Simulated biases for ML and PQL estimates under misspecified Gaussian random effects for GLMM's, but the random effects are autocorrelated: $\operatorname{cov}\left(u_{i j}, u_{i k}\right)=\sigma^{2} \rho^{|j-k|}$.

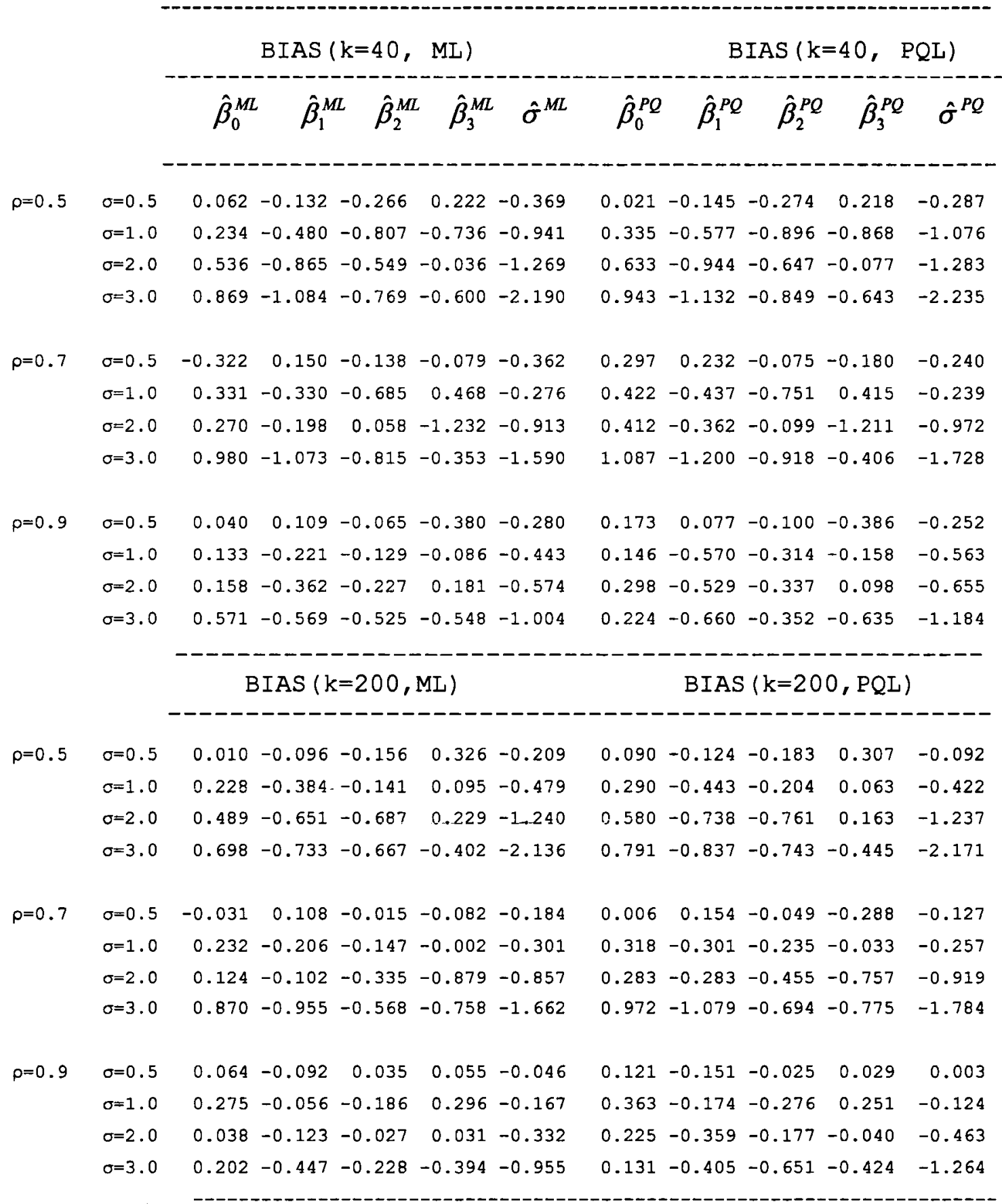




\section{Table 4.2:}

Root mean squared error for $M L$ and $P Q L$ estimates under misspecified Gaussian random effects for GLMM's, but the random effects are autocorrelated: $\operatorname{cov}\left(u_{i j}, u_{i k}\right)=\sigma^{2} \rho^{|j-k|}$.

\begin{tabular}{|c|c|c|c|c|c|c|c|c|c|c|c|}
\hline & & \multicolumn{5}{|c|}{$\operatorname{RootMSE}(k=40, M L)$} & \multicolumn{5}{|c|}{ RootMSE ( $k=40$, PQL $)$} \\
\hline & & $\hat{\beta}_{0}^{M L}$ & $\hat{\beta}_{1}^{M L}$ & $\hat{\beta}_{2}^{M L}$ & $\hat{\beta}_{3}^{M L}$ & $\hat{\sigma}^{M L}$ & $\hat{\beta}_{0}^{P Q}$ & $\hat{\beta}_{1}^{P Q}$ & $\hat{\beta}_{2}^{P Q}$ & $\hat{\beta}_{3}^{P Q}$ & $\hat{\sigma}^{P Q}$ \\
\hline \multirow[t]{4}{*}{$\rho=0.5$} & $\sigma=0.5$ & 0.428 & 0.837 & 0.722 & 1.146 & 0.408 & 0.410 & 0.825 & 0.718 & 1.148 & 0.359 \\
\hline & $\sigma=1.0$ & 0.583 & 0.949 & 1.024 & 1.280 & 0.739 & 0.756 & 0.598 & 1.176 & 1.432 & 0.732 \\
\hline & $\sigma=2.0$ & 0.743 & 1.186 & 0.969 & 0.925 & 1.332 & 0.779 & 1.195 & 0.983 & 0.886 & 1.338 \\
\hline & $\sigma=3.0$ & 0.938 & 1.365 & 1.185 & 0.959 & 2.272 & 0.995 & 1.382 & 0.816 & 0.945 & 2.297 \\
\hline \multirow[t]{4}{*}{$\rho=0.7$} & $\sigma=0.5$ & 0.723 & 0.573 & 0.939 & 1.310 & 0.462 & 0.737 & 0.246 & 0.952 & 1.311 & 0.448 \\
\hline & $\sigma=1.0$ & 0.884 & 0.829 & 1.421 & 1.365 & 0.331 & 0.877 & 0.834 & 1.395 & 1.290 & 0.285 \\
\hline & $\sigma=2.0$ & 0.675 & 0.675 & 0.981 & 1.500 & 0.959 & 0.638 & 0.677 & 0.907 & 1.447 & 1.006 \\
\hline & $\sigma=3.0$ & 1.072 & 1.215 & 1.092 & 1.309 & 1.661 & 1.108 & 1.296 & 1.132 & 1.223 & 1.868 \\
\hline \multirow[t]{6}{*}{$p=0.9$} & $\sigma=0.5$ & 0.290 & 0.319 & 0.453 & 0.492 & 0.415 & 0.254 & 0.314 & 0.413 & 0.501 & 0.395 \\
\hline & $\sigma=1.0$ & 0.311 & 0.594 & 0.476 & 0.704 & 0.644 & 0.316 & 0.607 & 0.488 & 0.806 & 0.637 \\
\hline & $\sigma=2.0$ & 0.365 & 0.683 & 0.500 & 0.985 & 0.778 & 0.397 & 0.693 & 0.531 & 1.824 & 0.793 \\
\hline & $\sigma=3.0$ & 0.818 & 0.875 & 0.973 & 1.305 & 1.056 & 0.932 & 0.917 & 0.957 & 1.233 & 1.288 \\
\hline & & $\mathrm{R}$ & ootMSE & $(k=20$ & $0, \mathrm{ML})$ & . & - & Root & $\operatorname{ISE}(\mathrm{k}$ & $00, \mathrm{PC}$ & ) \\
\hline & & ---- & 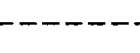 & --- & ---- & ---- & - & --- & ---- & ---- & --- \\
\hline \multirow[t]{4}{*}{$\rho=0.5$} & $\sigma=0.5$ & 0.300 & 0.476 & 0.410 & 0.996 & 0.285 & 0.318 & 0.496 & 0.422 & 0.975 & 0.163 \\
\hline & $\sigma=1.0$ & 0.343 & 0.472 & 0.376 & 0.651 & 0.503 & 0.385 & 0.519 & 0.397 & 0.616 & 0.442 \\
\hline & $\sigma=2.0$ & 0.507 & 0.687 & 0.857 & 0.761 & 1.250 & 0.591 & 0.767 & 0.899 & 0.707 & 1.245 \\
\hline & $\sigma=3.0$ & 0.769 & 1.330 & 0.685 & 0.915 & 2.150 & 0.886 & 1.312 & 0.792 & 0.829 & 2.183 \\
\hline \multirow[t]{4}{*}{$\rho=0.7$} & $\sigma=0.5$ & 0.438 & 0.266 & 0.760 & 0.823 & 0.296 & 0.419 & 0.529 & 0.737 & 0.809 & 0.298 \\
\hline & $\sigma=1.0$ & 0.343 & 0.463 & 0.387 & 0.588 & 0.321 & 0.400 & 0.495 & 0.407 & 0.564 & 0.283 \\
\hline & $\sigma=2.0$ & 0.529 & 0.536 & 0.413 & 0.532 & 0.874 & 0.549 & 0.554 & 0.510 & 0.518 & 0.933 \\
\hline & $\sigma=3.0$ & 1.008 & 1.063 & 0.678 & 1.113 & 1.395 & 1.031 & 1.162 & 0.776 & 1.073 & 1.804 \\
\hline \multirow[t]{4}{*}{$p=0.9$} & $\sigma=0.5$ & 0.123 & 0.162 & 0.289 & 0.469 & 0.247 & 0.161 & 0.209 & 0.267 & 0.458 & 0.261 \\
\hline & $\sigma=1.0$ & 0.516 & 0.313 & 0.409 & 0.666 & 0.270 & 0.600 & 0.356 & 0.442 & 0.632 & 0.226 \\
\hline & $\sigma=2.0$ & 0.295 & 0.521 & 0.469 & 0.484 & 0.413 & 0.366 & 0.583 & 0.548 & 0.440 & 0.513 \\
\hline & $\sigma=3.0$ & 0.584 & 0.774 & 0.745 & 1.219 & 1.025 & 0.925 & 0.915 & 0.803 & 1.108 & 1.224 \\
\hline
\end{tabular}


Here we investigate the effects of violation of random effects assumptions on estimation when random intercepts are assumed for data generated with autoregressive random effects for cluster of size $n=5$ and $k=(40,200)$. As before, a series of 500 data sets were generated for each set of parameter values of $\sigma$ and $\rho$, with $\beta$ using fixed at $\beta=(-2,2,2,1)$. Table 4.1 presents simulated biases for the ML and PQL estimates of $\beta$ and $\sigma$ that result when random intercepts are assumed for data generated with autoregressive random effects. We observe that when $\sigma$ is small $(0.5$ or 1.0$)$, the PQL method provides smaller biases in $\hat{\sigma}$, but the biases in the PQL estimates $\hat{\beta}^{P Q}$ are generally larger as compared to the ML estimate $\hat{\beta}^{M L}$ in most situations.

Table 4.2 shows corresponding simulated root mean squared errors for $\hat{\beta}$ and $\hat{\sigma}$ when random intercepts are assumed for data generated with autoregressive random effects. Here we observe that when the random effects have weak autocorrelation, (that is, when $\rho$ and $\sigma$ are small), the ML method provides smaller mean squared error in most situations. For stronger autocorrelation, the root mean squared errors in both $M L$ and $P Q L$ estimates are increased substantially, but the ML method provides more efficient estimates as compared to the PQL method. For example, when $\mathrm{k}=200, \rho=0.9$ and $\sigma=3.0$, the ML method provides root MSEs of $(0.584,0.774,0.745,1.219,1.025)$ for $\left(\hat{\beta}_{0}, \hat{\beta}_{1}, \hat{\beta}_{2}, \hat{\beta}_{3}\right)$ and the PQL method provides the corresponding values as $(0.925,0.915,0.803,01.108,1.224)$. 


\section{Chapter 6}

\section{Conclusion}

The purpose of the thesis was to investigate the impact of model violations on the estimates of regression coefficients in a generalized linear mixed model (GLMM). We evaluated the bias and MSE that resulted from incorrect assumptions regarding random effects. We focused on the logistic regression models for clustered binary response data. The simulation results indicate that use of a misspecified generalized linear mixed model for the estimation of the covariate effects can result in seriously biased regression estimates. The model misspecification also increases the mean squared errors of the estimates. In some situation, when the extern of model misspecification is large, the PQL method appears to provide more robust estimates of regression-coefficients in terms of smaller bias and mean squared error as compared to the maximum likelihood method. But there is no clear indication that the PQL method performs better for all situations where random effects assumptions are incorrectly specified. So careful attention should be given to the random effects model assumptions when using generalized linear mixed models for regression inference with clustered or longitudinal correlated data. 


\section{Appendix: R codes used for Simulation Study}

A1: $R$ codes when Random Intercepts are Non-Gaussian.

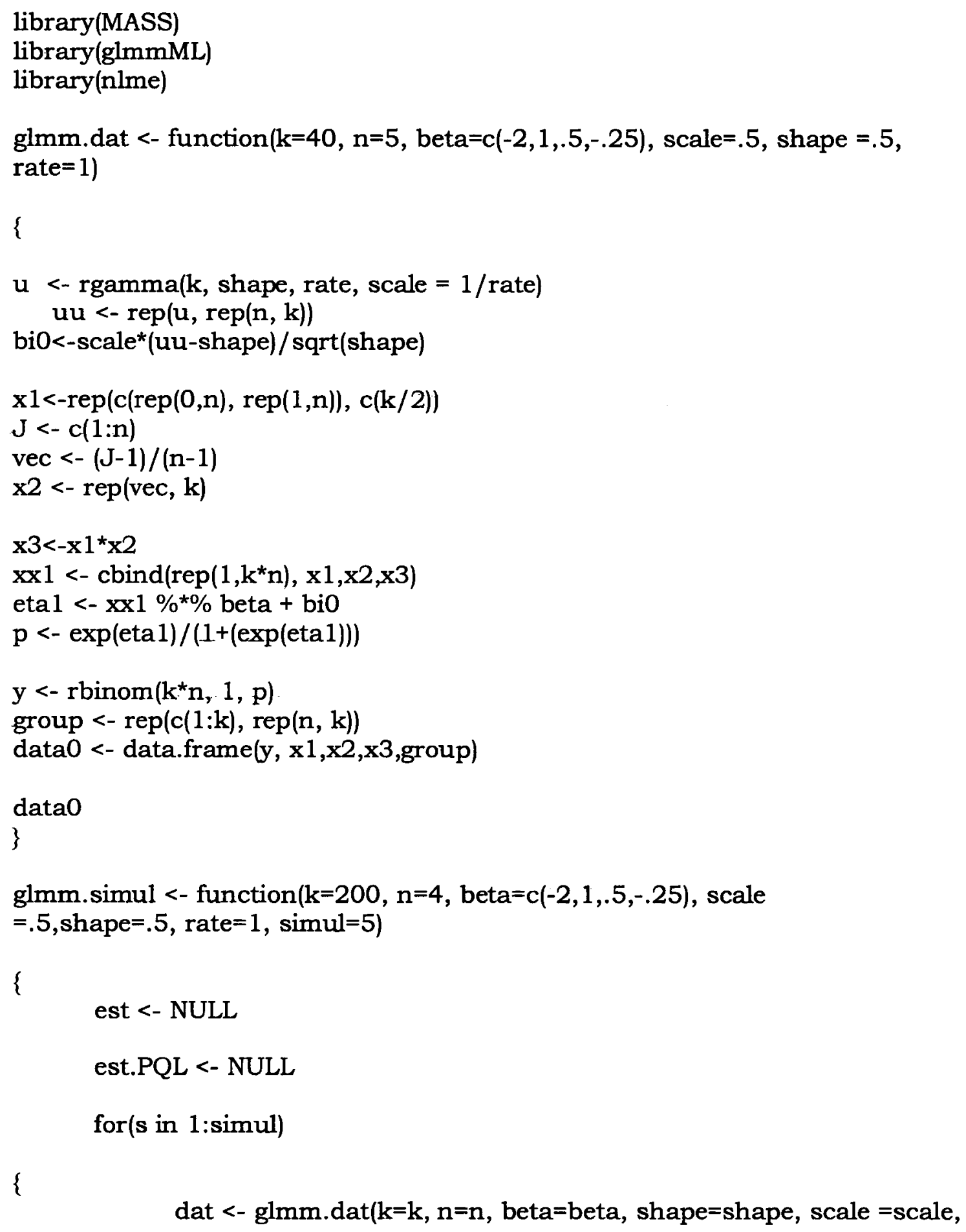




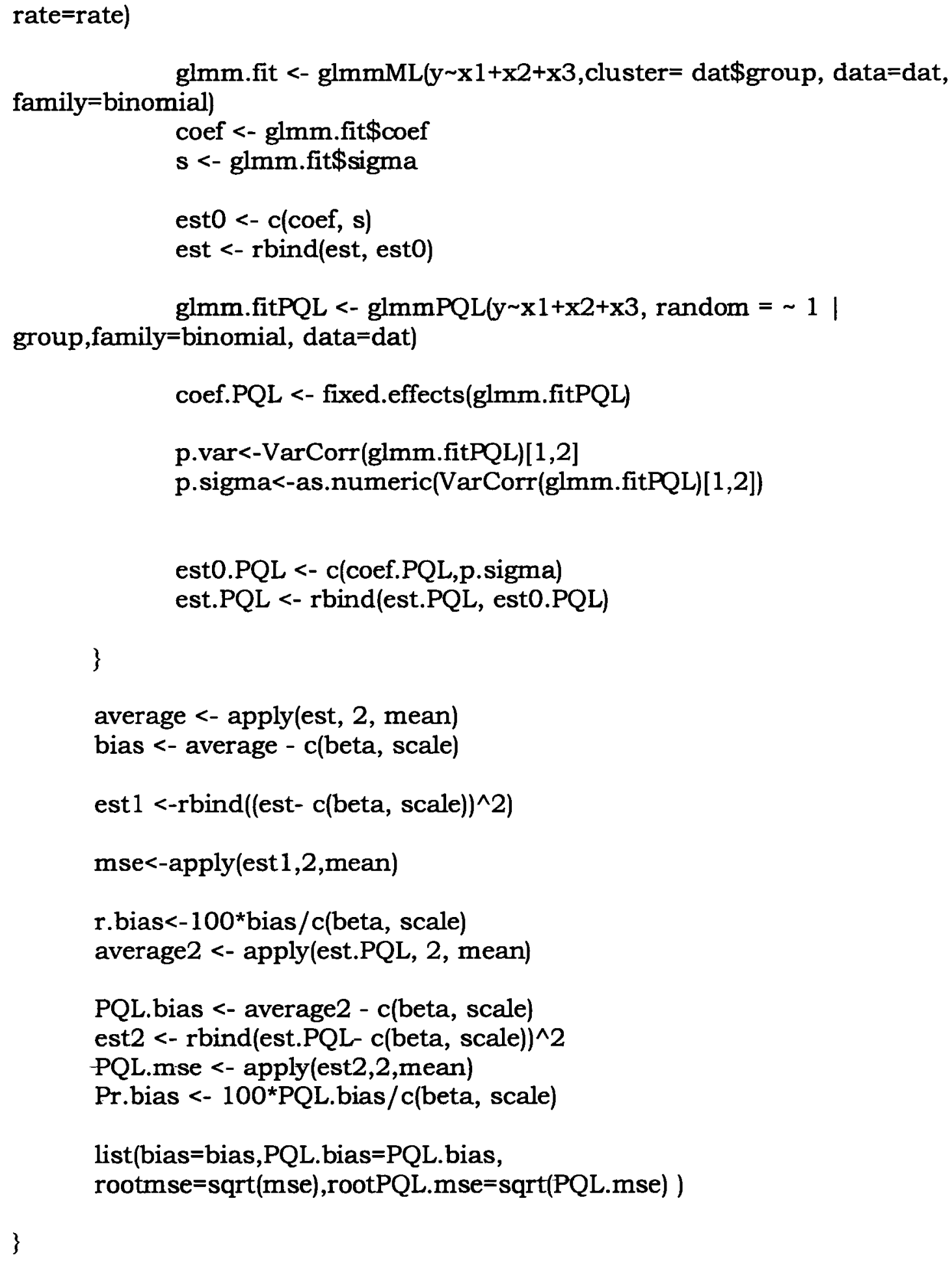




\section{References}

Abramowitz, M. and Stegun, I. (1964), "Hamdbook of Mathematical Functions", National Bureau of Standards, Washing, D. C.

Akaike, H. (1973), "Information Theory and an Extension of the Likelihood Principle, in Processing of the Second International Symposium of Information theory", B. N. Petrov and F. Caski, Budapest: Akademiai Kiado.

Azzalini, A. (1994), "Logistic Regression for Autocorrelated Data with Application to Repeated Measures", Biometrika, 81:767-775.

Berk, R. H. (1970), "Consistency of Posteriori", Annals of Mathematical Statistics, 41:894-906.

---- (1966), "Limiting Behavior of Posterior Distributions When the Model is Incorrect", Annals of Mathematical statistics", 37:51-58.

Breslow, N.E., and Clayton, D. G. (1993),"Approximate Inference in Generalized Linear Mixed Models", Journal of American Statistical Association, 88:9-25.

Cox, D. R. and Reid, N. (1987), "Parameter Orthogonality and Approximate Conditional Inference”, Journal of Royal Statistical Society, 49:1-39.

Durbin, J., and Koopman, S. J. (1997), "Monte Carlo maximum likelihood estimation for non-Gaussian state space models", Biometrika, 84:669-684.

Fisher, R.A. (1925), "Theory of Estimation", Proceedings of the Cambridge Philosophical Society, 22:700-725.

---- (1922), "On the Mathematical Foundations of Theoretical Statistics", Philosophical Transactions of the Royal Society of London, Series $A$, 222:309-368.

Gelfand, A., Carlin, B. (1993), "Maximum-likelihood estimation for constrainedor missing-data models", Canadian Joumal of Statistics, 21:303-311. 
Geyer, A., and Thompson, E. A. (1992), "Constrained Monte Carlo maximum likelihood for dependent data", Journal of Royal Statistical Society, Series B, 54:657-699.

Green, P. J., Silverman, B. W. (1994), “Nonparametric Regression and Generalized Linear Models”, Chapman \& Hall, London.

Harville, D. A. (1977), “Matrix Algebra from a Statistician's Perspective, Springer-Verlag, New York.

Heagerty, P. J., and Zeger, S. L. (2000), "Marginalized Multilevel Models for Longitudinal Binary Data", Statistical Science, 15:1-26.

Heagerty, P.J. (2000), " Marginal Multivariate Model and Likelihood Inference (with discussion)", Statistica Science, 15:1-26.

Heagerty, P.J., and Kurland, B. F. (2001), "Misspecified Maximum Likelihood Estimates and Generalized Linear Mixed Models", Biometrica, 88(4):973-985.

Huber, P.J. (1967), "The Behavior of maximum Likelihood Estimates under Nonstandard Conditions, in processing of the Fifth Berkeley Symposium in Mathematical Statistics and probability, Berkeley", University of California Press.

Kullback, S., and R. A. Leibler, R. A. (1951), "On Information and Sufficiency", Annals of Mathematical Statistics, 22:79-86

LeCam, L. (1953), "On some Asymptotic Properties of Maximum Likelihood Estimates and Related Bayes Estimates", University of California Publications in Statistics 1:277-330.

Liang, K.Y., and Hanfelt, J. (1994), "On the use of Quasi-Likelihood Method in Teratological Experiments", Biometrics, 50:872-80

Liang, K. Y., and Zeger, S. L. (1988), "Analysis of Longitudinal Data", Oxford Statistical Science Series. Second Edition.

--.- (1986), "Longitudinal Data Analysis using Generalized Linear Models", Biometrika, 73:13-22.

Longford, N.T., (1993), "Random Coefficient Models", Oxford University Press.

McCulloch, C. H., and Searle, S. R. (2001), "Generalized, Linear, and Mixed Models", Wiley Series in Probability and Statistics. John Wiley and Sons, Inc., 605, Third Avenue, New York, 10158-0012.

McCulloch, C. E. (1994), "Maximum Likelihood variance Components Estimation for Binary Data", Journal of American Statistical Association, 89:330-335. 
Nelder, J. A. and Wedderburn, R. W. M (1972), “ Generalized linear models,”. Journal of the Royal Statistical Society, Series A (General), 135(3):370-- 384.

Neuhaus, J. M., Hauck, W. W., and Kalbfleisch, J. D. (1992). "The Effects of Mixture Distribution Misspecification when Fitting Mixed-Effect Logistic Models", Biometrica, 79:755-762.

Palta, M., Lin, C. H. M., and Chao, W. H. (1997), "Effect of Confounding and other Misspecification in Models for Longitudinal Data", Lectures Notes in Statistics, Springer-Verlag, Berlin, 122:77-87

Patterson, H. D. and Thonpson, R. (1971), "Recovery of inter-block information when block sizes are unequal", Biometrika, 58:545-554

Rotnitzky, A., and Wypij, D., (1994), "A Note on the Basis of Estimators with Missing Data", Biometrics, 50:1163-1170.

Schall, R. (1991), "Estimation In Generalized Linear Models with random Effects", Biometrica, 80:791-795.

Sinha, S. K. (2004), "Robust Analysis of Generalized Linear Mixed Models", Journal of American Statistical Association, Vol 99, Number 466.

Sutradhar, B.C., and Sinha, S. K. (2002), "On Pseudo-Likelihood Inference in the Binary Longitudinal Mixed Model," Communication in Statistics, Part- $A$ Theory and Methods, 31, 397-417

Tan, M., Qu, Y., and Rao, J. S. (1999), "Robustness of the Latent Variable Model for Correlated Binary Data", Biometrics, 55:258-63.

Ten Have, T. R., Kunselman, A. R. and Tran, L. (1999), “ A comparison of mixed effects logistic regression models for binary response data with two nested levels of clustering," Statist. Med. 18:947-60

Tierney, L. and Kadane, J. B. (1986), "Accurate approximation for posterior moments and marginal densities", Journal of American Statistical Association, $81: 82-86$

Wald, A. (1943), "Tests of Statistical Hypothesis Concerning Several Parameters When the Number of Observations is Large", Transactions of the American Mathematical Society, 54:426-482.

Wald, A. (1949), " Note on the Consistency of the Maximum Likelihood Estimate", Annals of Mathematical Statistics, 60:595-603.

Wedderburn, R. W. M (1974), "Quasi-likelihood functions, generalized linear models, and the Gauss-Newton method," Biometrika, 61: 439-447. 
White, H. (1982), " Maximum Likelihood Estimates of Misspecified Models". Econometrica, 50:1-26.

White, H. (1980), "Using Least Squares to Approximate Unknown Regression Functions", International Economic Review, 21:149-170.

White, H. (1980), "Nonlinear Regression on Cross-Section Data", Econometrica, 48:817-838

Zeger, S. L., Lianh, K. Y., and Albert, P. S. (1988), "Models for Longitudinal Data: A Generalized Estimating Equation Approach", Biometrics, 44:10491060 